\title{
No-Go Theorem for Linear Systems on Bounded Bandlimited Signals
}

\author{
Holger Boche, Senior Member, IEEE, Ullrich J. Mönich, Student Member, IEEE, Andreas Kortke, and \\ Wilhelm Keusgen
}

\begin{abstract}
In this paper we analyze the existence of efficient bandpass-type systems for the space of bounded bandlimited signals. Here efficient means that the system fulfills the following properties: every output signal contains only frequencies within the passband; every input signal that has only frequencies within the passband is not disturbed by the system; and the system is stable. Without using any further assumptions, such as time-invariance, we prove that a linear realization cannot exist. Moreover, we show that a nonlinear realization is possible. It is well-known that every signal with finite energy can be split into two signals with finite energy, each of which contains a different part of the spectrum. Surprisingly, this does not hold for the space of bounded bandlimited signals. It is shown that there exist bounded bandlimited signals that cannot be split in the above way. These results can be of relevance for all applications where filters are used and the peak value of the signals is decisive, e.g., the design of efficient power amplifiers in wireless communication systems. The no-go results in this paper are helpful to better understand the signal space of bounded bandlimited signals and the limits of signal processing operations on this space.
\end{abstract}

Index Terms-Bandpass, frequency splitting, linear realization, lowpass, nonlinear realization, stability, system.

\section{INTRODUCTION}

$\mathbf{F}$ ILTERS are widely used in signal processing and system theory. The filtering of signals is especially descriptive when the signals are treated in the frequency domain. Loosely speaking, filters can be used to extract certain frequency portions from the signal, while other frequencies are suppressed. The passband of a filter is a certain frequency interval that specifies the behavior of the filter. All signals that have only frequencies within the passband are not disturbed by the filter. Filters can be characterized according to their passband. It is common to distinguish between lowpass-type, highpass-type, bandpass-type, and bandstop-type filters.

Manuscript received December 18, 2009; accepted June 16, 2010. Date of publication July 12, 2010; date of current version October 13, 2010. The associate editor coordinating the review of this manuscript and approving it for publication was Dr. Soontorn Oraintara. This work was supported in part by the German Federal Ministry of Economics and Technology by grant BMWi 01MT07007. The material in this paper was presented in part at the 2010 IEEE International Conference on Acoustics, Speech, and Signal Processing.

H. Boche was with the Technische Universität Berlin, Heinrich-Hertz-Chair for Mobile Communications, Einsteinufer 25, D-10578 Berlin, Germany. He is now with Technische Universität München, 80333 München, Germany (e-mail: holger.boche@mk.tu-berlin.de).

U. J. Mönich and A. Kortke are with the Technische Universität Berlin, Heinrich-Hertz-Chair for Mobile Communications, Einsteinufer 25, D-10578 Berlin, Germany (e-mail: ullrich.moenich@mk.tu-berlin.de; andreas.kortke@mk.tuberlin.de).

W. Keusgen is with the Fraunhofer Institut für Nachrichtentechnik, HeinrichHertz-Institut, Berlin 10587, Germany (e-mail: keusgen@hhi.fraunhofer.de).

Digital Object Identifier 10.1109/TSP.2010.2057249
In wireless communication systems different transmission techniques are used, for example time-division multiple access (TDMA) in GSM systems and orthogonal frequency division multiplexing (OFDM) in the downlink of 3GPP long term evolution (LTE) systems. Depending on the employed transmission technique, different signal spaces are appropriate for the mathematical treatment of the corresponding communication system. While in TDMA systems the transmitted signal has a constant envelope at each symbol time, the peak value of the transmitted signal in OFDM systems depends crucially on the data which shall be transmitted, and can get large compared to the average signal power. A common measure to quantify the peakiness of an OFDM signal is the peak-to-average power ratio (PAPR) [1]. OFDM systems are spectrally efficient but the transmit signals exhibit large PAPR values in general. For signals with large PAPR value it difficult and expensive to build high-efficiency power amplifiers. Thus, compared to traditional GSM systems, where the power amplifier design poses no problem, it is much more difficult to built efficient power amplifiers for LTE systems which use OFDM. Consequently, in order to save energy and costs, it is desirable to have OFDM transmit signals with small a PAPR value. Since, assuming a constant average signal power, the PAPR value of a signal depends on the peak value of the signal, it is natural to use the signal space $\mathcal{B}_{\pi}^{\infty}$ of bounded and bandlimited signals equipped with the supremum norm for the analysis. A precise mathematical definition $\mathcal{B}_{\pi}^{\infty}$ will be given in the next section.

The reason for the large PAPR values in OFDM systems is rooted in the structure of the signals and thus lies in the nature of the signal space $\mathcal{B}_{\pi}^{\infty}$. The main objective of this paper is to better understand the space $\mathcal{B}_{\pi}^{\infty}$ and to identify the possible and the impossible signal processing operations for this space. In particular, we want to analyze whether certain filtering operations exist and how they can be realized.

In the following, we use the term system instead of filter because filters are often assumed to be linear and time-invariant, and we do not want to restrict our analysis a priori to systems with those properties.

For several reasons bandpass-type systems should be efficient in the sense that

P1) every output signal has only frequencies within the passband;

P2) every input signal that has only frequencies within the passband is not disturbed by the system; and

P3) the system is stable.

In many applications such efficient systems are desirable. For example in wireless communication systems the transmitted signal must be concentrated within certain predefined frequency 
bands. The fraction of the signal power outside these intervals has to be below some threshold, in order that the signal does not interfere with other services that use these frequencies. Ideally, the out-of-band power would be zero, i.e., the employed bandpass-type system, which is used to filter the signal, should fulfill property $\mathrm{P} 1$ ). On the other hand the desired signal within the passband should not be disturbed by the filter. This is what property P2) expresses. In property P3) we mean with stability that the norm of the output signal can be controlled.

It is reasonable to call a bandpass-type system ideal if, in addition to $\mathrm{P} 1)-\mathrm{P} 3$ ) it has the property that

P4) the system output is the zero signal for every signal that has no frequencies within the passband.

Obviously, the class of ideal bandpass-type systems is a subclass of the efficient bandpass-type systems. Note that the above definitions do not assume the systems to be time-invariant or linear. This definition of an ideal bandpass-type system corresponds to the accepted definition of an ideal bandpass-type filter [2], if we further assume that the system is linear.

Lowpass-type, highpass-type, bandpass-type, and bandstoptype systems are also frequently used in theoretical analyses. In [3] for example, Logan develops a theory of modulation systems, in which bandpass-type systems play a central role. Modulation systems are often based on the Hilbert transform. However, for general bounded bandlimited signals the Hilbert transform does not necessarily exist. Interestingly, for every bounded bandpass signal the Hilbert transform exists and is bounded [4].

The main results of this paper about the realizability and existence of signals processing operations on the space $\mathcal{B}_{\pi}^{\infty}$ of bounded and bandlimited signals, given in Sections IV, V, and VI, are as follows. In Section IV we prove that there exists no linear (not even a time-variant) realization of efficient bandpasstype systems for the signal space $\mathcal{B}_{\pi}^{\infty}$, and in Section V we show that a nonlinear realization of efficient bandpass-type systems exists. Moreover, in Section VI we prove that a splitting of the signals with respect to their frequency content, is not possible in general. As a consequence, two operations, which are often used and whose existence is generally taken for granted, the extraction of frequency components and the splitting of a signal into two parts with disjoint spectrum, cannot be performed in a stable manner for the signal space $\mathcal{B}_{\pi}^{\infty}$.

\section{NOTATION AND DEFINITIONS}

In order to continue, we need some notation and definitions. Let $\hat{f}$ denote the Fourier transform of a function $f . L^{p}(\mathbb{R})$, $1 \leq p<\infty$, is the space of all $p$ th-power Lebesgue integrable functions on $\mathbb{R}$, with the usual norm $\|\cdot\|_{p}$, and $L^{\infty}(\mathbb{R})$ is the space of all functions for which the essential supremum norm $\|\cdot\|_{\infty}$ is finite. $L^{p}[a, b], 1 \leq p \leq \infty$, is the space of all functions in $L^{p}(\mathbb{R})$ whose support is in the closed interval $[a, b] . C_{0}(\mathbb{R})$ denotes the space of all continuous functions on $\mathbb{R}$ that vanish at infinity, and $C_{0}^{\infty}[a, b]$ the space of all infinitely differentiable functions on $\mathbb{R}$ whose support is in $[a, b]$.

For $0<\sigma<\infty$ let $\mathcal{B}_{\sigma}$ be the set of all entire functions $f$ with the property that for all $\epsilon>0$ there exists a constant $C(\epsilon)$ with $|f(z)| \leq C(\epsilon) \exp ((\sigma+\epsilon)|z|)$ for all $z \in \mathbb{C}$. The Bernstein space $\mathcal{B}_{\sigma}^{p}, 1 \leq p \leq \infty$, consists of all functions in
$\mathcal{B}_{\sigma}$, whose restriction to the real line is in $L^{p}(\mathbb{R})$. The norm for $\mathcal{B}_{\sigma}^{p}$ is given by the $L^{p}$-norm on the real line, i.e., $\|\cdot\|_{\mathcal{B}_{\sigma}^{p}}=$ $\|\cdot\|_{p}$. A signal in $\mathcal{B}_{\sigma}^{p}$ is called bandlimited to $\sigma$, and $\mathcal{B}_{\sigma}^{\infty}$ is the space of bounded and bandlimited signals. For signals in $\mathcal{B}_{\sigma}^{\infty}$, we have the following lemma, which is a consequence of the Phragmén-Lindelöf principle [5, Lecture 6].

Lemma 1: Let $f \in \mathcal{B}_{\sigma}^{\infty}, \sigma>0$. Then we have $|f(z)| \leq$ $\|f\|_{\infty} \mathrm{e}^{\sigma|\operatorname{Im}(z)|}$ for all $z \in \mathbb{C}$.

Moreover, $\mathcal{B}_{\sigma, 0}^{\infty}$ denotes the set of all signals in $\mathcal{B}_{\sigma}^{\infty}$ that vanish at infinity. An important property of the space $\mathcal{B}_{\sigma, 0}^{\infty}$ is stated in the following lemma, the proof of which is given in Appendix A.

Lemma 2: The normed space $\left(\mathcal{B}_{\sigma, 0}^{\infty},\|\cdot\|_{\infty}\right), 0<\sigma<\infty$, is complete.

For $0<\sigma<\infty$ and $1 \leq p \leq \infty$ we denote by $\mathcal{P} \mathcal{W}_{\sigma}^{p}$ the Paley-Wiener space of signals $f$ with a representation $f(z)=$ $1 /(2 \pi) \int_{-\sigma}^{\sigma} g(\omega) \mathrm{e}^{i z \omega} \mathrm{d} \omega, z \in \mathbb{C}$, for some $g \in L^{p}[-\sigma, \sigma]$. If $f \in \mathcal{P} \mathcal{W}_{\sigma}^{p}$ then $g(\omega)=\hat{f}(\omega)$. The norm for $\mathcal{P} \mathcal{W}_{\sigma}^{p}, 1 \leq p<\infty$, is given by $\|f\|_{\mathcal{P} \mathcal{W}_{\sigma}^{p}}=\left(1 /(2 \pi) \int_{-\sigma}^{\sigma}|\hat{f}(\omega)|^{p} \mathrm{~d} \omega\right)^{1 / p}$. For $p=2$ we obtain the Paley-Wiener space $\mathcal{P} \mathcal{W}_{\sigma}^{2}$, which is nothing else than the space of bandlimited signals with finite energy.

As we can see from the above definitions, all signals in $\mathcal{B}_{\sigma}^{p}$ and in $\mathcal{P} \mathcal{W}_{\sigma}^{p}, 0<\sigma<\infty, 1 \leq p \leq \infty$, are defined on the complex plane. However, in practical applications the signals are usually considered to be a function of a real variable, which often represents the time. Since all signals in the above spaces are entire functions, they are uniquely determined by their values on the real line. Therefore, we will not distinguish between signals defined on the complex plane and signals defined on the real axis in the following. For example, if $f$ is a function defined on the real axis and we write $f \in \mathcal{B}_{\sigma}^{p}$, we mean that $f$ can be extended to an entire function, defined on the complex plane, which is in $\mathcal{B}_{\sigma}^{p}$. Equally, if $f$ is an entire function and we write $f \in L^{p}(\mathbb{R})$, we mean that the restriction of $f$ to the real axis is in $L^{p}(\mathbb{R})$.

\section{A. An Alternative Definition of Bandlimitedness}

For signals in $\mathcal{B}_{\pi}^{\infty}$ the Fourier transform does not need to exist. Hence, we have to be careful when we use terms like "frequencies of a signal". However, it will turn out that-with the proper definitions - we can mathematically rigorously formulate the properties P1)-P3) of efficient bandpass-type systems, which were listed in an intuitive way in Section I, for systems operating on $\mathcal{B}_{\pi}^{\infty}$.

We use the following definition to declare bounded bandpass signals. This definition is similar to Zakai's definition of bandlimited signals in [6]. For $-\infty<\omega_{1}<\omega_{2}<\infty$ let

$$
\mathcal{K}_{+}\left(\omega_{1}, \omega_{2}\right)=\left\{f \in L^{1}(\mathbb{R}): \hat{f}(\omega)=1 \text { for } \omega \in\left[\omega_{1}, \omega_{2}\right]\right\} .
$$

The space $\mathcal{B}_{+\left[\omega_{1}, \omega_{2}\right]}^{\infty}$ consists of all signals $f \in L^{\infty}(\mathbb{R})$ that fulfill $f(t)=\int_{-\infty}^{\infty} f(\tau) K(t-\tau) \mathrm{d} \tau$ for all $t \in \mathbb{R}$ and all $K \in$ $\mathcal{K}_{+}\left(\omega_{1}, \omega_{2}\right)$. The norm of $\mathcal{B}_{+\left[\omega_{1}, \omega_{2}\right]}$ is given by the $L^{\infty}$-norm on the real line.

The following lemma shows that bounded bandlimited signals are a special case of bounded bandpass signals. Hence, this definition of bandlimitedness is closely related to the one given in the previous section.

Lemma 3: Let $\sigma>0$. Then we have $\mathcal{B}_{+[-\sigma, \sigma]}^{\infty}=\mathcal{B}_{\sigma}^{\infty}$. 
The proof of Lemma 3 is given in Appendix B. Furthermore, $\mathcal{B}_{+\left[\omega_{1}, \omega_{2}\right]}^{\infty}$ has the important property to be complete. We will use this fact in the proof of Theorem 2.

Lemma 4: Let $-\infty<\omega_{1}<\omega_{2}<\infty$ and $\sigma=$ $\max \left\{\left|\omega_{1}\right|,\left|\omega_{2}\right|\right\}$. Then $\mathcal{B}_{+\left[\omega_{1}, \omega_{2}\right]}^{\infty}$ is a closed subspace of $\mathcal{B}_{\sigma}^{\infty}$ and complete.

The proof of Lemma 4 is included in Appendix C.

Analogously to the one-sided bandpass signals, we define the two-sided bandpass signals. For $0 \leq \omega_{1}<\omega_{2}<\infty$ let

$$
\mathcal{K}\left(\omega_{1}, \omega_{2}\right)=\left\{f \in L^{1}(\mathbb{R}): \hat{f}(\omega)=1 \text { for }|\omega| \in\left[\omega_{1}, \omega_{2}\right]\right\} .
$$

The space $\mathcal{B}_{\left[\omega_{1}, \omega_{2}\right]}^{\infty}$ consists of all signals $f \in L^{\infty}(\mathbb{R})$ that fulfill $f(t) \stackrel{\left[\omega_{1}, \omega_{2}\right.}{=} \int_{-\infty}^{\infty} f(\tau) K(t-\tau) \mathrm{d} \tau$ for all $t \in \mathbb{R}$ and all $K \in \mathcal{K}\left(\omega_{1}, \omega_{2}\right)$.

Note that, according to the previous definitions and Lemma 3 , we have $\mathcal{B}_{[0, \sigma]}^{\infty}=\mathcal{B}_{+[-\sigma, \sigma]}^{\infty}=\mathcal{B}_{\sigma}^{\infty}$. Without loss of generality we can restrict the analyses to the bandwidth $\sigma=\pi$. Any other bandwidth can be obtained by a simple scaling of the signal.

\section{B. LTI Systems}

We briefly review some definitions and facts about stable LTI systems. A linear system $T: \mathcal{A} \rightarrow \mathcal{B}$, mapping signals from the space $\mathcal{A}$ to the space $\mathcal{B}$, is called stable if the operator $T$ is bounded, i.e., if $\|T\|=\sup _{\|f\|_{\mathcal{A}} \leq 1}\|T f\|_{\mathcal{B}}<\infty$. Furthermore, it is called time invariant if $(\operatorname{Tf}(\cdot-a))(t)=(T f)(t-a)$ for all $f \in \mathcal{A}$ and $t, a \in \mathbb{R}$.

Remark 1: Note that our definition of stability is with respect to the norms of the spaces $\mathcal{A}$ and $\mathcal{B}$, and thus is different from the concept of bounded input—-bounded output (BIBO) stability in general.

Mathematically, a system is an operator, i.e., a rule by which an input signal is transformed into an output signal. This operator can have different representations. For example, one possible representation for stable LTI systems operating on signals in $\mathcal{P W}_{\pi}^{2}$ is the following well-known frequency-domain representation. For every stable LTI system $T: \mathcal{P} \mathcal{W}_{\pi}^{2} \rightarrow \mathcal{P W}_{\pi}^{2}$ there exists exactly one function $\hat{h}_{T} \in L^{\infty}[-\pi, \pi]$ such that

$$
(T f)(t)=\frac{1}{2 \pi} \int_{-\pi}^{\pi} \hat{f}(\omega) \hat{h}_{T}(\omega) \mathrm{e}^{i \omega t} \mathrm{~d} \omega
$$

for all $f \in \mathcal{P} \mathcal{W}_{\pi}^{2}$ and all $t \in \mathbb{R}$. The operator norm of $T$ is given by $\|T\| \stackrel{\pi}{=}\left\|\hat{h}_{T}\right\|_{\infty}$ and the impulse response $h_{T}$ by $h_{T}=T$ sinc. Conversely, every function $\hat{h}_{T} \in L^{\infty}[-\pi, \pi]$ defines a stable LTI system $T: \mathcal{P} \mathcal{W}_{\pi}^{2} \rightarrow \mathcal{P} \mathcal{W}_{\pi}^{2}$. Thus, the space of all stable LTI systems defined on $\mathcal{P} \mathcal{W}_{\pi}^{2}$ is isometrically isomorphic to $L^{\infty}[-\pi, \pi]$. Note that $\hat{h}_{T} \in L^{\infty}[-\pi, \pi] \subset L^{2}[-\pi, \pi]$ and consequently $h_{T} \in \mathcal{P} \mathcal{W}_{\pi}^{2}$. Another possible representation for stable LTI systems operating on signals in $\mathcal{P W}_{\pi}^{2}$ is the following time-domain representation in the form of a convolution integral. For every stable LTI system $T: \mathcal{P W}_{\pi}^{2} \rightarrow \mathcal{P W} \mathcal{W}_{\pi}^{2}$ we have

$$
(T f)(t)=\int_{-\infty}^{\infty} f(\tau) h_{T}(t-\tau) \mathrm{d} \tau
$$

for all $f \in \mathcal{P W}_{\pi}^{2}$ and all $t \in \mathbb{R}$.

\section{Stability Concepts}

A common concept of stability is bounded input—bounded output (BIBO) stability.

Definition 1: A LTI system $T: L^{\infty}(\mathbb{R}) \rightarrow L^{\infty}(\mathbb{R})$ is called BIBO-stable if

$$
\sup _{\substack{f \in L \infty(\mathbb{R}) \\\|f\|_{\infty} \leq 1}}\|T f\|_{\infty}<\infty .
$$

According to this definition, the output of every BIBO-stable LTI system is bounded. Furthermore, for bounded and bandlimited signals we define the following notion of stability.

Definition 2: A LTI system $T: \mathcal{B}_{\pi, 0}^{\infty} \rightarrow L^{\infty}(\mathbb{R})$ is called $\mathrm{BIBO}_{\pi}$-stable if

$$
\sup _{\substack{f \in \mathcal{B}_{\pi, 0}^{\infty} \\\|f\|_{\mathcal{B}}^{\infty} \leq, \leq 1}}\|T f\|_{\infty}<\infty .
$$

Using the common notation, we denote by

$$
\|T\|_{\mathrm{BIBO}_{\pi}}:=\sup _{\substack{f \in \mathcal{B}_{\pi, 0}^{\infty} \\\|f\|_{\mathcal{B}_{\pi, 0}^{\infty} \leq 1}^{\infty} \leq}}\|T f\|_{\infty}
$$

the operator norm of $T$.

From both definitions above and the fact that $\mathcal{B}_{\pi, 0}^{\infty} \subset L^{\infty}(\mathbb{R})$, it follows immediately that every BIBO-stable LTI system is also a $\mathrm{BIBO}_{\pi}$-stable LTI system.

The next lemma gives a necessary and sufficient condition for the $\mathrm{BIBO}_{\pi}$-stability of a LTI system.

Lemma 5: Let $T: \mathcal{B}_{\pi, 0}^{\infty} \rightarrow L^{\infty}(\mathbb{R})$ be a LTI system. $T$ is $\mathrm{BIBO}_{\pi}$-stable if and only if for all $\omega_{1}>\pi$ there exists a $h \in \mathcal{B}_{\omega_{1}}^{1}$ such that

$$
(T f)(t)=\int_{-\infty}^{\infty} f(\tau) h(t-\tau) \mathrm{d} \tau
$$

for all $f \in \mathcal{B}_{\pi, 0}^{\infty}$ and all $t \in \mathbb{R}$.

In the proof, which is given in Appendix $\mathrm{D}$, we will see that the sufficient condition for $\mathrm{BIBO}_{\pi}$-stability can be further weakened. A LTI system $T: \mathcal{B}_{\pi, 0}^{\infty} \rightarrow L^{\infty}(\mathbb{R})$ is $\mathrm{BIBO}_{\pi}$-stable if for some $\omega_{1}>\pi$ there exists a $h \in \mathcal{B}_{\omega_{1}}^{1}$ such that (3) is true for all $f \in \mathcal{B}_{\pi, 0}^{\infty}$.

\section{REALIZATION OF SYSTEMS}

In an abstract view, an efficient system is an operator that maps every input signal to an output signal in accordance with the above properties P1)-P3). It is important to distinguish between the abstract concept of a system, or more general of an operator, and the actual realization. An operator can have many possible realizations with different properties. To illustrate these differences we give two examples.

The differential operator is well defined on the space of bounded bandlimited signals that vanish at infinity. One realization of this operator is given by

$$
f^{\prime}(t)=\sum_{k=-\infty}^{\infty} f(k)(-1)^{k} \frac{\pi(t-k) \cos (\pi t)-\sin (\pi t)}{\pi(t-k)^{2}} .
$$


This series is formally obtained from the Shannon sampling series

$$
f(t)=\sum_{k=-\infty}^{\infty} f(k) \frac{\sin (\pi(t-k))}{\pi(t-k)}
$$

by differentiating termwise. Another possible realization, which is obtained by differentiating the Valiron interpolation series [7], [8, p. 12]

$f(t)=f\left(\frac{1}{2}\right) \sin (\pi t)+\left(t-\frac{1}{2}\right) \sum_{k=-\infty}^{\infty} \frac{f(k)}{k-\frac{1}{2}} \frac{\sin (\pi(t-k))}{\pi(t-k)}$

is given by

$$
\begin{aligned}
f^{\prime}(t)=f\left(\frac{1}{2}\right) & \pi \cos (\pi t)+\sum_{k=-\infty}^{\infty} \frac{\sin (\pi(t-k)) f(k)}{\pi(t-k)\left(k-\frac{1}{2}\right)} \\
& \times\left(1+\left(t-\frac{1}{2}\right) \frac{(t-k) \cot (\pi t) \pi-1}{t-k}\right) .
\end{aligned}
$$

The two realizations have different properties. For example, the sum in the second realization (5) is absolutely convergent, while the sum in the first realization (4) is not absolutely convergent in general. Thus, from the fact that one realization does not have a specific property we cannot conclude that there exist no realization with this property.

In order to further illustrate what we mean by realization of a system, we consider again the differential operator and ask whether a certain system with prescribed properties exists and how a realization of this system looks like. Let $C_{b}$ denote the space of complex-valued bounded and continuous signals defined on $\mathbb{R}$ with the norm $\|f\|_{C_{b}}=\sup _{t \in \mathbb{R}}|f(t)|$. Can we find a system $D$ that maps every signal in $C_{b}$ to a bandlimited signal, additionally has the property that $\sup _{\|f\|_{C_{b}} \leq 1}|(D f)(t)|<\infty$ for all $t \in \mathbb{R}$, and fulfills $D f=f^{\prime}$ for all $f \in \mathcal{B}_{\pi}^{\infty}$ ? Thus, the system is defined on the large signal space $C_{b}$ and equals the differential operator on the subspace $\mathcal{B}_{\pi}^{\infty}$. For signals in $\mathcal{B}_{\pi}^{\infty}$, the differential operator is well defined and we have $\left\|f^{\prime}\right\|_{\infty} \leq$ $\pi\|f\|_{\infty}$ according to Bernstein's inequality [9, p. 49].

A first attempt to get a realization of the desired system $D$ is to use a realization of the differential operator which is valid for signals in the space $\mathcal{P} \mathcal{W}_{\pi}^{2} \subset C_{b}$. For signals $f \in \mathcal{P} \mathcal{W}_{\pi}^{2}$ we have

$$
f(t)=\int_{-\infty}^{\infty} f(\tau) \frac{\sin (\pi(t-\tau))}{\pi(t-\tau)} \mathrm{d} \tau
$$

and $f^{\prime}(t)=\left(D^{1} f\right)(t)$, where

$$
\left(D^{1} f\right)(t):=\int_{-\infty}^{\infty} f(\tau) \frac{\cos (\pi(t-\tau)) \pi(t-\tau)-\sin (\pi(t-\tau))}{\pi(t-\tau)^{2}} \mathrm{~d} \tau .
$$

We see that (6) is a linear and time-invariant realization of the differential operator for signals in $\mathcal{P W}_{\pi}^{2}$. Although (6) is a possible realization of the differential operator for $\mathcal{P} \mathcal{W}_{\pi}^{2}$, it is no realization of the desired system $D$, because

$$
\sup _{\|f\|_{C_{b} \leq 1}}\left|\left(D^{1} f\right)(0)\right|=\infty
$$

which means that the operator $D^{1}$ is not stable for $C_{b}$. Equation (7) can verified by showing that $\left(D^{1} f_{1}\right)(0)=\infty$ for the signal

$$
f_{1}(t)= \begin{cases}0, & |t|<\pi / 2 \\ \cos (\pi t), & |t| \geq \pi / 2 .\end{cases}
$$

However, the divergence of (6) for certain signals in $C_{b}$ does not imply that the desired system $D$ does not exist. For example, a possible time-variant realization of the desired system $D$ is given by (5).

In this paper we analyze the existence of bandpass-type systems operating on the space $\mathcal{B}_{\pi}^{\infty}$ of bounded and bandlimited signals. It will turn out that it is impossible to find a linear realization of efficient bandpass-type systems because linear efficient bandpass-type systems system do not exist for the space $\mathcal{B}_{\pi}^{\infty}$. Surprisingly, a nonlinear realization is possible.

\section{NO-Go THEOREM FOR LINEAR REALIZATION OF EFFICIENT BANDPASS-TYPE SYSTEMS}

In this section we analyze whether a linear realization of efficient bandpass-type systems exists for $\mathcal{B}_{\pi}^{\infty}$, i.e., the space of bounded bandlimited signals.

Theorem 1: Let $0 \leq \omega_{1}<\omega_{2} \leq \pi$ with $w_{2}-w_{1}<\pi$. There exists no linear operator $T$ defined on $\mathcal{B}_{\pi}^{\infty}$ with the properties

i) $\operatorname{range}(T) \subseteq \mathcal{B}_{\left[\omega_{1}, \omega_{2}\right]}^{\infty}$;

ii) $T f=f$ for all $f \in \mathcal{B}_{\left[\omega_{1}, \omega_{2}\right]}^{\infty}$;

iii) $T: \mathcal{B}_{\pi}^{\infty} \rightarrow \mathcal{B}_{\left[\omega_{1}, \omega_{2}\right]}^{\infty}$ is bounded.

The properties i)-iii) are the mathematical formulation of the properties P1)-P3), which determine an efficient system. Property i) means that the system output has only frequencies in the range $\left[-\omega_{2},-\omega_{1}\right] \cup\left[\omega_{1}, \omega_{2}\right]$. Property ii) says that every signal with frequencies concentrated in the passband is not disturbed by the system, and property iii) expresses the stability of the system. The additional condition $w_{2}-w_{1}<\pi$ in Theorem 1 was only included to prevent the case where $\omega_{1}=0$ and $\omega_{2}=\pi$. In this case the identity operator would trivially fulfill the properties i), ii), and iii).

Theorem 1 shows that there exists no linear operator defined on $\mathcal{B}_{\pi}^{\infty}$ with the properties i)-iii). Consequently, a linear realization of efficient bandpass-type systems for the signal space $\mathcal{B}_{\pi}^{\infty}$ cannot exist. Since the class of ideal bandpass-type systems is a subclass of the efficient bandpass-type systems, this implies that there exists no linear realization of ideal bandpass-type systems.

Remark 2: Since every lowpass-type system can be seen as a bandpass-type system with a passband that starts from zero, Theorem 1 also implies that there exists no linear realization of efficient lowpass-type systems for the signal space $\mathcal{B}_{\pi}^{\infty}$.

Note that the result of Theorem 1 is very general, because there are many conceivable realizations. For example we do not restrict the systems to be time-invariant.

Proof of Theorem 1: The proof is divided into two parts. In the fist part we prove the assertion for $0 \leq \omega_{1}<\omega_{2}<\pi$ and in the second part for $0<\omega_{1}<\omega_{2}=\pi$.

First part: Let $\omega_{1}, \omega_{2} \in \mathbb{R}$ with $0 \leq \omega_{1}<\omega_{2}<\pi$ be arbitrary but fixed. The non-existence is proved indirectly. We assume that there exists a bounded linear operator $T$ defined on $\mathcal{B}_{\pi}^{\infty}$ with the properties i) and ii) and construct a contradiction. 
For the proof we need the functions

$$
f_{N}(t)=\frac{\mathrm{e}^{i \omega_{2} t}}{2 i} \sum_{k=1}^{\lfloor N \gamma\rfloor} \frac{1}{k}\left(\mathrm{e}^{i \pi \frac{k}{N} t}-\mathrm{e}^{-i \pi \frac{k}{N} t}\right), \quad N \in \mathbb{N}
$$

where

$$
\gamma=\min \left\{\frac{\omega_{2}-\omega_{1}}{\pi}, 1-\frac{\omega_{2}}{\pi}\right\} .
$$

Thus, $f_{N} \in \mathcal{B}_{\pi}^{\infty}$ for all $N \in \mathbb{N}$. Moreover, let $f_{N, \tau}(t)=f_{N}(t+$ $\tau)$. Then we have

$$
\left\|f_{N, \tau}\right\|_{\infty}=\left\|f_{N}\right\|_{\infty}=\left\|\sum_{k=1}^{\lfloor N \gamma\rfloor} \frac{\sin \left(\frac{\pi k}{N} \cdot\right)}{k}\right\|_{\infty} \leq C_{1}<\infty
$$

for all $N \in \mathbb{N}$ and all $\tau \in \mathbb{R}$, where the constant $C_{1}$ is independent of $N$ [10, p. 183]. For the moment, let $N \in \mathbb{N}$ and $t \in \mathbb{R}$ be arbitrary but fixed. We have

$$
\begin{aligned}
\left(T f_{N, \tau}\right)(t-\tau) & =\frac{1}{2 i} \sum_{k=1}^{\lfloor N \gamma\rfloor} \frac{1}{k} \mathrm{e}^{i \tau\left(\omega_{2}+\frac{k}{N} \pi\right)} \underbrace{T\left[\mathrm{e}^{i \cdot\left(\omega_{2}+\frac{k}{N} \pi\right)}\right](t-\tau)}_{=: u(k, \tau, t)} \\
& -\frac{1}{2 i} \sum_{k=1}^{\lfloor N \gamma\rfloor} \frac{1}{k} \mathrm{e}^{i \tau\left(\omega_{2}-\frac{k}{N} \pi\right)} T\left[\mathrm{e}^{i \cdot\left(\omega_{2}-\frac{k}{N} \pi\right)}\right](t-\tau) .
\end{aligned}
$$

Since $\omega_{2}-(k / N) \pi \geq \omega_{1}$ for all $1 \leq k \leq\lfloor N \gamma\rfloor$, the function $\mathrm{e}^{i \cdot\left(\omega_{2}-(k / N) \pi\right)}$ is in $\mathcal{B}_{\left[\omega_{1}, \omega_{2}\right]}^{\infty}$ and it follows that

$$
T\left[\mathrm{e}^{i \cdot\left(\omega_{2}-\frac{k}{N} \pi\right)}\right](t-\tau)=\mathrm{e}^{i(t-\tau)\left(\omega_{2}-\frac{k}{N} \pi\right)}
$$

for all $1 \leq k \leq\lfloor N \gamma\rfloor$, according to property ii), which is assumed to be true. Further, we know from i) that $u(k, \cdot, t) \in$ $\mathcal{B}_{\omega_{2}}^{\infty}$ for $1 \leq k \leq\lfloor N \gamma\rfloor$. Consequently, we obtain

$$
\begin{aligned}
\left(T f_{N, \tau}\right)(t-\tau)= & \frac{1}{2 i} \sum_{k=1}^{\lfloor N \gamma\rfloor} \frac{1}{k} \mathrm{e}^{i \tau\left(\omega_{2}+\frac{k}{N} \pi\right)} u(k, \tau, t) \\
& -\frac{1}{2 i} \sum_{k=1}^{\lfloor N \gamma\rfloor} \frac{1}{k} \mathrm{e}^{i \tau\left(\omega_{2}-\frac{k}{N} \pi\right)} \mathrm{e}^{i(t-\tau)\left(\omega_{2}-\frac{k}{N} \pi\right)} \\
= & \frac{1}{2 i} \sum_{k=1}^{\lfloor N \gamma\rfloor} \frac{1}{k} \mathrm{e}^{i \tau\left(\omega_{2}+\frac{k}{N} \pi\right)} u(k, \tau, t) \\
& -\frac{1}{2 i} \sum_{k=1}^{\lfloor N \gamma\rfloor} \frac{1}{k} \mathrm{e}^{i t\left(\omega_{2}-\frac{k}{N} \pi\right)}
\end{aligned}
$$

and it follows that

$$
\begin{aligned}
\frac{1}{2 M} \int_{-M}^{M}\left(T f_{N, \tau}\right)(t-\tau) \mathrm{d} \tau \\
=\frac{1}{2 i} \sum_{k=1}^{\lfloor N \gamma\rfloor} \frac{1}{k} \frac{1}{2 M} \int_{-M}^{M} \mathrm{e}^{i \tau\left(\omega_{2}+\frac{k}{N} \pi\right)} u(k, \tau, t) \mathrm{d} \tau \\
\quad-\frac{1}{2 i} \sum_{k=1}^{\lfloor N \gamma\rfloor} \frac{1}{k} \mathrm{e}^{i t\left(\omega_{2}-\frac{k}{N} \pi\right)} .
\end{aligned}
$$

Next, we treat the integral on the right-hand side (RHS) of (9) for $1 \leq k \leq\lfloor N \gamma\rfloor$. According to Cauchy's integral theorem, we have

$$
\oint_{P_{M}^{(1)} \cup P_{M}^{(2)}} \mathrm{e}^{i z\left(\omega_{2}+\frac{k}{N} \pi\right)} u(k, z, t) \mathrm{d} z=0 .
$$

where $P_{M}^{(1)}$ and $P_{M}^{(2)}$ are the integration paths depicted in Fig. 1. Since $u(k, \cdot, t) \in \mathcal{B}_{\omega_{2}}^{\infty}$, Lemma 1 guarantees the existence of a constant $C_{2}(k, t)$ such that

$$
|u(k, z, t)| \leq C_{2}(k, t) \mathrm{e}^{\omega_{2}|\operatorname{Im}(z)|}
$$

for all $z \in \mathbb{C}$, and it follows that

$$
\begin{aligned}
& \left|\frac{1}{2 M} \int_{-M}^{M} \mathrm{e}^{i \tau\left(\omega_{2}+\frac{k}{N} \pi\right)} u(k, \tau, t) \mathrm{d} \tau\right| \\
& \quad=\left|\frac{1}{2 M} \int_{P_{M}^{(1)}} \mathrm{e}^{i z\left(\omega_{2}+\frac{k}{N} \pi\right)} u(k, z, t) \mathrm{d} z\right| \\
& \quad \leq \frac{1}{2} \int_{0}^{\pi}\left|\mathrm{e}^{i M \mathrm{e}^{i \phi}\left(\omega_{2}+\frac{k}{N} \pi\right)}\right|\left|u\left(k, M \mathrm{e}^{i \phi}, t\right)\right| \mathrm{d} \phi \\
& \leq \frac{C_{2}(k, t)}{2} \int_{0}^{\pi} \mathrm{e}^{-M \sin (\phi)\left(\omega_{2}+\frac{k}{N} \pi\right)} \mathrm{e}^{\omega_{2} M \sin (\phi)} \mathrm{d} \phi \\
& \quad=\frac{C_{2}(k, t)}{2} \int_{0}^{\pi} \mathrm{e}^{-\frac{k}{N} \pi M \sin (\phi)} \mathrm{d} \phi .
\end{aligned}
$$

Bearing in mind that

$$
\int_{0}^{\pi / 2} \mathrm{e}^{-\frac{k}{N} \pi M \sin (\phi)} \mathrm{d} \phi=\int_{\pi / 2}^{\pi} \mathrm{e}^{-\frac{k}{N} \pi M \sin (\phi)} \mathrm{d} \phi
$$

and that $\sin (\phi) \geq 2 \phi / \pi$ for all $\phi \in[0, \pi / 2]$, we can further evaluate the integral on the RHS of (10)

$$
\begin{aligned}
\frac{C_{2}(k, t)}{2} \int_{0}^{\pi} \mathrm{e}^{-\frac{k}{N} \pi M \sin (\phi)} \mathrm{d} \phi & \leq C_{2}(k, t) \int_{0}^{\pi / 2} \mathrm{e}^{-\frac{2 k M}{N} \phi} \mathrm{d} \phi \\
& =C_{2}(k, t) \frac{N}{2 k M}\left(1-\mathrm{e}^{-\frac{k M \pi}{N}}\right) .
\end{aligned}
$$

Thus, we obtain

$$
\begin{aligned}
\mid \frac{1}{2 M} \int_{-M}^{M} \mathrm{e}^{i \tau\left(\omega_{2}+\frac{k}{N} \pi\right)} u(k, \tau, t) & \mathrm{d} \tau \mid \\
& \leq C_{2}(k, t) \frac{N}{2 k M}\left(1-\mathrm{e}^{-\frac{k M \pi}{N}}\right)
\end{aligned}
$$

and consequently

$$
\lim _{M \rightarrow \infty} \frac{1}{2 M} \int_{-M}^{M} \mathrm{e}^{i \tau\left(\omega_{2}+\frac{k}{N} \pi\right)} u(k, \tau, t) \mathrm{d} \tau=0
$$


for all $1 \leq k \leq\lfloor N \gamma\rfloor$. Using (11), it follows from (9) that

$\lim _{M \rightarrow \infty}\left|\frac{1}{2 M} \int_{-M}^{M}\left(T f_{N, \tau}\right)(t-\tau) \mathrm{d} \tau\right|=\left|\frac{1}{2} \sum_{k=1}^{\lfloor N \gamma\rfloor} \frac{1}{k} \mathrm{e}^{i t\left(\omega_{2}-\frac{k}{N} \pi\right)}\right|$.

Since $T$ is assumed to be a bounded linear operator, we have $\|T\|=\sup _{\|f\|_{\mathcal{B}^{\infty} \leq 1} \leq 1}\|T f\|_{\infty}<\infty$, and $\|T f\|_{\infty} \leq\|T\|\|f\|_{\infty}$ for all $f \in \mathcal{B}_{\pi}^{\infty}$. It follows that

$$
\begin{aligned}
\left|\frac{1}{2 M} \int_{-M}^{M}\left(T f_{N, \tau}\right)(t-\tau) \mathrm{d} \tau\right| & \leq \frac{1}{2 M} \int_{-M}^{M}\left|\left(T f_{N, \tau}\right)(t-\tau)\right| \mathrm{d} \tau \\
& \leq\left\|T f_{N, \tau}\right\|_{\infty} \leq\|T\|\left\|f_{N, \tau}\right\|_{\infty} \\
& \leq\|T\| C_{1}<\infty
\end{aligned}
$$

Thus, from (12) and (13) we obtain

$$
\left|\frac{1}{2} \sum_{k=1}^{\lfloor N \gamma\rfloor} \frac{1}{k} \mathrm{e}^{i t\left(\omega_{2}-\frac{k}{N} \pi\right)}\right| \leq\|T\| C_{1}<\infty
$$

which is valid for all $N \in \mathbb{N}$ and $t \in \mathbb{R}$, because $N$ and $t$ were arbitrary. On the other hand, since

$$
\lim _{N \rightarrow \infty} \frac{1}{2} \sum_{k=1}^{\lfloor N \gamma\rfloor} \frac{1}{k} \geq \frac{1}{2} \lim _{N \rightarrow \infty} \log (N \gamma)=\infty
$$

we see that the left-hand side (LHS) of (14) diverges for $t=0$ as $N$ tends to infinity. This is a contradiction.

Second part: Let $\omega_{1} \in \mathbb{R}$ with $0<\omega_{1}<\omega_{2}=\pi$ be arbitrary but fixed, and assume that there is an bounded linear operator $T$ defined on $\mathcal{B}_{\pi}^{\infty}$ with the properties i) and ii). Let $M_{\sigma}, \sigma \in$ $\mathbb{R}$, denote the operator $\left(M_{\sigma} f\right)(t)=\mathrm{e}^{i \sigma t} f(t)$. Furthermore, let $k_{1} \in \mathcal{K}_{+}\left(\omega_{1}, \pi\right)$, and define the operator

$$
\left(T_{1} f\right)(t)=\left(f * k_{1}\right)(t)=\int_{-\infty}^{\infty} f(\tau) k_{1}(t-\tau) \mathrm{d} \tau .
$$

We have that $T_{1} f \in \mathcal{B}_{+\left[\omega_{1}, \pi\right]}^{\infty}$. Moreover, it follows from (15) that $\left\|T_{1} f\right\|_{\infty} \leq\|f\|_{\infty}^{+\omega}\left\|k_{1}\right\|_{L^{1}(\mathbb{R})}$. Thus, $T_{1}: \mathcal{B}_{\left[\omega_{1}, \pi\right]}^{\infty} \rightarrow$ $\mathcal{B}_{+\left[\omega_{1}, \pi\right]}^{\infty}$ is a bounded operator. Finally, we need the operator $U=M_{-\pi} T_{1} T M_{\pi}$. Clearly, $U$ is a bounded linear operator that maps $\mathcal{B}_{+[-\pi, 0]}^{\infty}$ onto $\mathcal{B}_{+\left[\omega_{1}-\pi, 0\right]}^{\infty}$. For all $f \in \mathcal{B}_{+\left[\omega_{1}-\pi, 0\right]}^{\infty}$ we have $T_{1} T M_{\pi} f=M_{\pi} f$ and consequently $U f \stackrel{\omega_{1}-\pi, 0}{=} M_{-\pi} T_{1} T M_{\pi} f=M_{-\pi} M_{\pi} f=f$. We can use the same proof technique as in part 1 to show that such an operator cannot exist.

\section{NONLINEAR REALIZATION OF EFFICIENT BANDPASS-TYPE SYSTEMS}

In this section we drop the condition that the system is linear. The following theorem shows that a nonlinear realization of efficient bandpass-type systems is possible for the space $\mathcal{B}_{\pi}^{\infty}$.

Theorem 2: Let $0 \leq \omega_{1}<\omega_{2} \leq \pi$. There exists an operator $T$ defined on $\mathcal{B}_{\pi}^{\infty}$ with the properties
i) $\operatorname{range}(T) \subseteq \mathcal{B}_{\left[\omega_{1}, \omega_{2}\right]}^{\infty}$;
ii) $T f=f$ for all $f \in \mathcal{B}_{\left[\omega_{1}, \omega_{2}\right]}^{\infty}$; and
iii) $\|T f\|_{\infty} \leq 2\|f\|_{\infty}$ for all $f \in \mathcal{B}_{\pi}^{\infty}$.

Theorem 2 states the existence of an operator with the properties i)-iii). However, it does not give a realization of this operator in the form of a closed-form expression. Nevertheless, the proof gives the details how an algorithm can be designed for the approximation of the operator.

Remark 3: Although Theorem 2 shows that a nonlinear realization of efficient bandpass-type systems is possible for the space $\mathcal{B}_{\pi}^{\infty}$, it makes no statement whether an ideal bandpasstype system can be realized.

Proof of Theorem 2: Let $f \in \mathcal{B}_{\pi}^{\infty} \backslash \mathcal{B}_{\left[\omega_{1}, \omega_{2}\right]}^{\infty}$ be arbitrary but fixed, and consider

$$
C\left(f, \omega_{1}, \omega_{2}\right)=\inf _{h \in \mathcal{B}_{\left[\omega_{1}, \omega_{2}\right]}^{\infty}}\|f-h\|_{\infty} .
$$

There exists a sequence $\left\{g_{n}\right\}_{n \in \mathbb{N}} \subset \mathcal{B}_{\left[\omega_{1}, \omega_{2}\right]}^{\infty}$ such that

$$
\lim _{n \rightarrow \infty}\left\|f-g_{n}\right\|_{\infty}=C\left(f, \omega_{1}, \omega_{2}\right) .
$$

Moreover, since $\left\|g_{n}\right\|_{\infty} \leq\left\|f-g_{n}\right\|_{\infty}+\|f\|_{\infty}$, there exists a natural number $n_{0}$ such that $\left\|g_{n}\right\|_{\infty} \leq 1+C\left(f, \omega_{1}, \omega_{2}\right)+\|f\|_{\infty}$ for all $n \geq n_{0}$. Thus, we can use Lemma 1 to obtain

$$
\begin{aligned}
\left|g_{n}(z)\right| & \leq\left\|g_{n}\right\|_{\infty} \mathrm{e}^{\omega_{2}|\operatorname{Im}(z)|} \\
& \leq\left(1+C\left(f, \omega_{1}, \omega_{2}\right)+\|f\|_{\infty}\right) \mathrm{e}^{\omega_{2}|\operatorname{Im}(z)|}
\end{aligned}
$$

for all $z \in \mathbb{C}$ and $n \geq n_{0}$. From Montel's theorem [11, p. 195] it follows that there exists an entire function $g$ and a subsequence $\left\{n_{k}\right\}_{k \in \mathbb{N}}$ such that $\lim _{k \rightarrow \infty} g_{n_{k}}(z)=g(z)$ for all $z \in \mathbb{C}$. Further, according to Lemma 4, we have $g \in \mathcal{B}_{\left[\omega_{1}, \omega_{2}\right]}^{\infty}$. Since $f \in \mathcal{B}_{\pi}^{\infty} \backslash \mathcal{B}_{\left[\omega_{1}, \omega_{2}\right]}^{\infty}$ was arbitrary, we can define an operator $T_{1}: \mathcal{B}_{\pi}^{\infty} \backslash \mathcal{B}_{\left[\omega_{1}, \omega_{2}\right]}^{\infty} \rightarrow \mathcal{B}_{\left[\omega_{1}, \omega_{2}\right]}^{\infty}, f \mapsto g$. On the other hand, for $f \in \mathcal{B}_{\left[\omega_{1}, \omega_{2}\right]}^{\infty}$ we define the operator $T_{2}: \mathcal{B}_{\left[\omega_{1}, \omega_{2}\right]}^{\infty} \rightarrow$ $\mathcal{B}_{\left[\omega_{1}, \omega_{2}\right]}^{\infty}, f \mapsto f$. Next, we show that the operator $T: \mathcal{B}_{\pi}^{\infty} \rightarrow$ $\mathcal{B}_{\left[\omega_{1}, \omega_{2}\right]}^{\infty}$ given by

$$
T f= \begin{cases}T_{1} f & \text { if } f \in \mathcal{B}_{\pi}^{\infty} \backslash \mathcal{B}_{\left[\omega_{1}, \omega_{2}\right]}^{\infty} \\ T_{2} f & \text { if } f \in \mathcal{B}_{\left[\omega_{1}, \omega_{2}\right]}^{\infty}\end{cases}
$$

is the desired operator with the properties i)-iii). The properties i) and ii) are obviously fulfilled, due to the construction of the operator $T$. Moreover, the inequality in iii) is true for all $f \in$ $\mathcal{B}_{\left[\omega_{1}, \omega_{2}\right]}^{\infty}$ because for these signals we have $\|T f\|_{\infty}=\|f\|_{\infty}$. It remains to prove the inequality in iii) for all $f \in \mathcal{B}_{\pi}^{\infty} \backslash \mathcal{B}_{\left[\omega_{1}, \omega_{2}\right]}^{\infty}$. For all $t \in \mathbb{R}$ we have $\left|f(t)-g_{n_{k}}(t)\right| \leq\left\|f-g_{n_{k}}\right\|_{\infty}$ and consequently

$$
\begin{aligned}
|f(t)-g(t)| & =\lim _{k \rightarrow \infty}\left|f(t)-g_{n_{k}}(t)\right| \\
& \leq \lim _{k \rightarrow \infty}\left\|f-g_{n_{k}}\right\|_{\infty} \\
& =C\left(f, \omega_{1}, \omega_{2}\right) .
\end{aligned}
$$

Taking the supremum on both sides of (18), we obtain $\| f-$ $g \|_{\infty} \leq C\left(f, \omega_{1}, \omega_{2}\right)$. This implies that

$$
\|f-g\|_{\infty}=C\left(f, \omega_{1}, \omega_{2}\right),
$$


because $C\left(f, \omega_{1}, \omega_{2}\right)=\inf _{h \in \mathcal{B}_{\left[\omega_{1}, \omega_{2}\right]}^{\infty}}\|f-h\|_{\infty} \leq\|f-g\|_{\infty}$. Equality (19) together with the fact that $C\left(f, \omega_{1}, \omega_{2}\right) \leq\|f\|_{\infty}$ gives

$$
\begin{aligned}
\|g\|_{\infty} & \leq\|f-g\|_{\infty}+\|f\|_{\infty} \\
& =C\left(f, \omega_{1}, \omega_{2}\right)+\|f\|_{\infty} \\
& \leq 2\|f\|_{\infty}
\end{aligned}
$$

which completes the proof.

\section{NO-Go THEOREM FOR FREQUeNCY SPLITTING}

In the previous section we have seen that a linear realization of efficient bandpass-type systems on $\mathcal{B}_{\pi}^{\infty}$ is impossible and that a nonlinear realization of efficient bandpass-type systems on $\mathcal{B}_{\pi}^{\infty}$ is possible. Although a nonlinear realization of efficient bandpass-type systems is possible it is still open whether ideal bandpass-type systems can be realized.

The material in this section is conceptually closely related to the previous section, especially to the idea of ideal bandpass-type systems. Suppose that ideal bandpass-type systems exist. Then, using two ideal bandpass-type systems, one with passband $\left[-\omega_{1}, \omega_{1}\right]$ and the other with passband $\left[-\pi,-\omega_{1}\right] \cup$ $\left[\omega_{1}, \pi\right], 0<\omega_{1}<\pi$, it would be possible, given any signal $f \in \mathcal{B}_{\pi}^{\infty}$, to generate two signals $f_{1} \in \mathcal{B}_{\left[0, \omega_{1}\right]}^{\infty}$ and $f_{2} \in \mathcal{B}_{\left[\omega_{1}, \pi\right]}^{\infty}$. If $f$ contains only frequencies in $\left[-\omega_{1}, \omega_{1}\right]$, i.e., if $f \in \mathcal{B}_{\left[0, \omega_{1}\right]}^{\infty}$, then $f_{1}$ equals $f$, and $f_{2}$ is the zero signal. In this case, we have $f=f_{1}+f_{2}$. However, in the more general case where $f \in \mathcal{B}_{\pi}^{\infty}$, we only know that $f_{1} \in \mathcal{B}_{\left[0, \omega_{1}\right]}^{\infty}$ and that $f_{2} \in \mathcal{B}_{\left[\omega_{1}, \pi\right]}^{\infty}$, but in general we do not have $f=f_{1}+f_{2}$. This is due to the very general axiomatic definition of ideal bandpass-type systems.

For the discussion so far, we only used the concept of bandpass signals. Next, we analyze whether a frequency splitting of signals $f \in \mathcal{B}_{\pi}^{\infty}$ into two signals $f_{1} \in \mathcal{B}_{\left[0, \omega_{1}\right]}^{\infty}$ and $f_{2} \in \mathcal{B}_{\left[\omega_{1}, \pi\right]}^{\infty}$, each of which contains a different part of the spectrum, is possible. Therefor we also need to define what it means that two signals agree on some frequency interval.

For bandlimited signals with finite energy, i.e., signals in $\mathcal{P} \mathcal{W}_{\pi}^{2}$, it is easy to define what it means that two signals $f$ and $g$ agree on some frequency interval $\left[\omega_{1}, \omega_{2}\right],-\pi<\omega_{1}<\omega_{2}<\pi$. This is done by comparing the Fourier transforms $\hat{f}$ and $\hat{g}$. If $\hat{f}(\omega)=\hat{g}(\omega)$ almost everywhere (a.e.) in $\left[\omega_{1}, \omega_{2}\right]$ we say that $f$ and $g$ agree on the frequency interval $\left[\omega_{1}, \omega_{2}\right]$. Note that it is irrelevant whether we include the endpoints of the intervals in the above definition or not, because a single point has Lebesgue measure zero. Thus, two signals in $\mathcal{P} \mathcal{W}_{\pi}^{2}$ agree on the closed frequency interval $\left[\omega_{1}, \omega_{2}\right]$ if and only if they agree on the open frequency interval $\left(\omega_{1}, \omega_{2}\right)$.

A nice property of the space $\mathcal{P} \mathcal{W}_{\pi}^{2}$ is that it is possible to split a signal with respect to its frequency content. For every $f \in \mathcal{P} \mathcal{W}_{\pi}^{2}$ and every frequency interval $\left[\omega_{1}, \omega_{2}\right],-\infty<\omega_{1}<$ $\omega_{2}<\infty$, we can find a signal $g \in \mathcal{P} \mathcal{W}_{\pi}^{2}$ that agrees with $f$ on the frequency interval $\left[\omega_{1}, \omega_{2}\right]$ and with the zero function on $[-\pi, \pi] \backslash\left[\omega_{1}, \omega_{2}\right]$. The signal $g$ is given by

$$
g(t)=\frac{1}{2 \pi} \int_{\omega_{1}}^{\omega_{2}} \hat{f}(\omega) \mathrm{e}^{i \omega t} \mathrm{~d} \omega .
$$

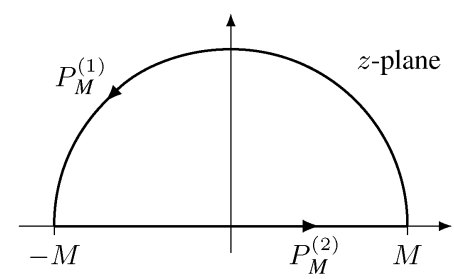

Fig. 1. Integration paths $P_{M}^{(1)}$ and $P_{M}^{(2)}$ in the complex plane.
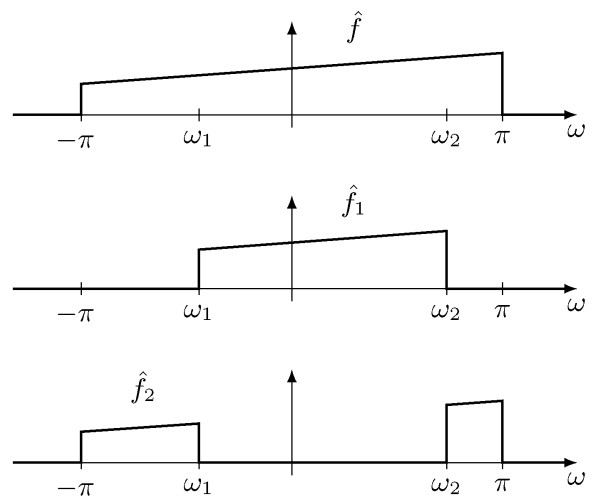

Fig. 2. Frequency splitting of a signal $f \in \mathcal{P} \mathcal{W}_{\pi}^{2}$ into two signals $f_{1} \in \mathcal{P} \mathcal{W}_{\pi}^{2}$ and $f_{2} \in \mathcal{P} \mathcal{W}_{\pi}^{2}$.

This consideration shows that for every signal $f \in \mathcal{P W}_{\pi}^{2}$ and every frequency interval $\left[\omega_{1}, \omega_{2}\right],-\infty<\omega_{1}<\omega_{2}<\infty$, it is possible to split $f$ into two signals $f_{1} \in \mathcal{P} \mathcal{W}_{\pi}^{2}$ and $f_{2} \in \mathcal{P} \mathcal{W}_{\pi}^{2}$ such that $f$ agrees with $f_{1}$ on the frequency interval $\left[\omega_{1}, \omega_{2}\right]$ and with $f_{2}$ on the frequency interval $[-\pi, \pi] \backslash\left[\omega_{1}, \omega_{2}\right]$. This frequency splitting is illustrated in Fig. 2. Due to the linearity of the Fourier transform we have $f=f_{1}+f_{2}$.

This way of splitting a signal with respect to its frequency content works for signals in $\mathcal{P W}_{\pi}^{2}$ and corresponds to the common way of thinking. Next, we analyze whether this splitting is possible for signals in $\mathcal{B}_{\pi}^{\infty}$, i.e., bounded and bandlimited signals. Since the Fourier transform does not necessarily exist for signals in $\mathcal{B}_{\pi}^{\infty}$, we cannot use the above way to define what it means that two signals in $\mathcal{B}_{\pi}^{\infty}$ agree on some frequency interval. Thus, we have to adapt the definition [12].

Definition 3: We say that $f \in \mathcal{B}_{\pi}^{\infty}$ and $g \in \mathcal{B}_{\pi}^{\infty}$ agree on the open frequency interval $\left(\omega_{1}, \omega_{2}\right),-\infty<\omega_{1}<\omega_{2}<\infty$, if

$$
\int_{-\infty}^{\infty} f(\tau) h(\tau) \mathrm{d} \tau=\int_{-\infty}^{\infty} g(\tau) h(\tau) \mathrm{d} \tau
$$

for all $h \in L^{1}(\mathbb{R})$ with $\hat{h}(\omega)=0$ for all $\omega \in \mathbb{R} \backslash\left(\omega_{1}, \omega_{2}\right)$.

We see that this definition makes only a statement about what it means that two signals agree on open sets of frequencies. It cannot be used to define what it means that two signals agree on a closed set of frequencies. For example, according to the above definition, the two signals $3 \sin (\pi t)$ and $5 \sin (\pi t)$ agree on every open interval $\left(-\omega_{1}, \omega_{1}\right), \omega_{1} \leq \pi$, and disagree on every open interval $\left(-\omega_{2}, \omega_{2}\right), \omega_{2}>\pi$. However, if we changed all open intervals to closed intervals in the above definition, the equality in (20) would still hold, because $\hat{h}$ is continuous and $\hat{h}(-\pi)=\hat{h}(\pi)=0$. This is a fundamental difference to the 


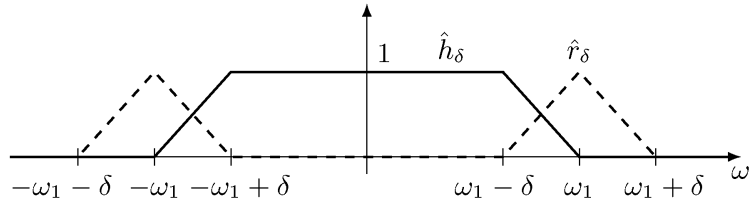

Fig. 3. Definition of $\hat{h}_{\delta}$ (solid line) and $\hat{r}_{\delta}$ (dashed line).

space $\mathcal{P} \mathcal{W}_{\pi}^{2}$, where we do not have to distinguish between open and closed sets in the definition.

This definition is reasonable, because for $f \in \mathcal{P W}_{\pi}^{2}$ it is equivalent to the definition that uses the Fourier transform.

Observation 1: Let $f, g \in \mathcal{P} \mathcal{W}_{\pi}^{2}$. We have $\hat{f}(\omega)=\hat{g}(\omega)$ a.e. in $\left[\omega_{1}, \omega_{2}\right]$ if and only if (20) holds for all $h \in L^{1}(\mathbb{R})$ with $\hat{h}(\omega)=0$ for all $\omega \in \mathbb{R} \backslash\left(\omega_{1}, \omega_{2}\right)$.

For completeness, we included the elementary proof of Observation 1 in Appendix E.

Equipped with this definition of two signals in $\mathcal{B}_{\pi}^{\infty}$ agreeing on some open interval of frequencies, we can ask the question: Given $0<\omega_{1}<\pi$, can we find for all signals $f \in \mathcal{B}_{\pi, 0}^{\infty}$ a decomposition $f=f_{1}+f_{2}$ with $f_{1} \in \mathcal{B}_{\omega_{1}}^{\infty}$ and $f_{2} \in \mathcal{B}_{\pi}^{\infty}$, such that $f$ and $f_{1}$ agree on the frequency interval $\left(-\omega_{1}, \omega_{1}\right)$ ? If this was possible it would immediately follow that $f_{2}$ agrees with the zero function on the frequency interval $\left(-\omega_{1}, \omega_{1}\right)$ and that $f_{2} \in \mathcal{B}_{\left[\omega_{1}, \pi\right]}^{\infty} . f_{1}$ would be the lowpass part of $f$, which agrees with $f$ on the open frequency interval $\left(-\omega_{1}, \omega_{1}\right)$, and $f_{2}$ would be the bandpass part of $f$, which agrees with $f$ on the open set of frequencies $\left(-\pi,-\omega_{1}\right) \cup\left(\omega_{1}, \pi\right)$.

In the following theorem we will see that a frequency splitting as discussed above is not possible for signals in $\mathcal{B}_{\pi}^{\infty}$. Although we restrict ourselves to the lowpass case in Theorem 3 , it is also true for the bandpass case.

Theorem 3: Let $0<\omega_{1}<\pi$. There exists a signal $f \in \mathcal{B}_{\pi, 0}^{\infty}$ such that there exists no signal $f_{\omega_{1}} \in \mathcal{B}_{\omega_{1}}^{\infty}$ such that

$$
\int_{-\infty}^{\infty} f(\tau) h(\tau) \mathrm{d} \tau=\int_{-\infty}^{\infty} f_{\omega_{1}}(\tau) h(\tau) \mathrm{d} \tau
$$

for all $h \in \mathcal{B}_{\omega_{1}}^{1}$.

Remark 4: Since $\mathcal{B}_{\pi, 0}^{\infty} \subset \mathcal{B}_{\pi}^{\infty}$, Theorem 3 remains true if we replace $\mathcal{B}_{\pi, 0}^{\infty}$ with $\mathcal{B}_{\pi}^{\infty}$.

Theorem 3 shows that the splitting of a signal into several signal components with disjoint spectrum, is not possible for the space $\mathcal{B}_{\pi}^{\infty}$. This signal theoretic result of course implies that there exists no filter-regardless of how complicated the realization is made- that can perform this task.

Proof of Theorem 3: We suppose that the negation of the assertion of Theorem 3 is true and construct a contradiction. Thus, we assume that there exists a $\omega_{1}$ with $0<\omega_{1}<\pi$ such that for all $f \in \mathcal{B}_{\pi, 0}^{\infty}$ there exists a signal $f_{\omega_{1}} \in \mathcal{B}_{\omega_{1}}^{\infty}$ such that (21) is true for all $h \in \mathcal{B}_{\omega_{1}}^{1}$.

Let $0<\delta<\omega_{1}$. For the proof we need the two functions $h_{\delta}$ and $r_{\delta}$, whose Fourier transforms $\hat{h}_{\delta}$ and $\hat{r}_{\delta}$ are depicted in Fig. 3. A simple calculation gives

$$
r_{\delta}(t)=\frac{2}{\pi \delta t^{2}} \cos \left(\omega_{1} t\right)(1-\cos (\delta t))
$$

and it follows that

$$
\begin{aligned}
\int_{-\infty}^{\infty}\left|r_{\delta}(t)\right| \mathrm{d} t & \leq \frac{2}{\pi} \int_{-\infty}^{\infty}\left|\frac{1-\cos (\delta t)}{\delta t^{2}}\right| \mathrm{d} t \\
& =\frac{2}{\pi} \int_{-\infty}^{\infty}\left|\frac{1-\cos (t)}{t^{2}}\right| \mathrm{d} t \\
& =2
\end{aligned}
$$

independently of $\delta$. According to our assumption we have, for all $f \in \mathcal{B}_{\pi, 0}^{\infty}$

$$
\begin{aligned}
\int_{-\infty}^{\infty} f(\tau) h_{\delta}(\tau) \mathrm{d} \tau= & \int_{-\infty}^{\infty} f_{\omega_{1}}(\tau) h_{\delta}(\tau) \mathrm{d} \tau \\
= & \int_{-\infty}^{\infty} f_{\omega_{1}}(\tau)\left(h_{\delta}(\tau)+r_{\delta}(\tau)\right) \mathrm{d} \tau \\
& -\int_{-\infty}^{\infty} f_{\omega_{1}}(\tau) r_{\delta}(\tau) \mathrm{d} \tau \\
= & f_{\omega_{1}}(0)-\int_{-\infty}^{\infty} f_{\omega_{1}}(\tau) r_{\delta}(\tau) \mathrm{d} \tau
\end{aligned}
$$

where the last equality follows from the fact that $h_{\delta}+r_{\delta} \in$ $\mathcal{K}\left(0, \omega_{1}\right)$ and $f \in \mathcal{B}_{\left[0, \omega_{1}\right]}^{\infty}$. Moreover, since

$$
\int_{-\infty}^{\infty} f_{\omega_{1}}(\tau) r_{\delta}(\tau) \mathrm{d} \tau \leq\left\|f_{\omega_{1}}\right\|_{\infty} \int_{-\infty}^{\infty}\left|r_{\delta}(t)\right| \mathrm{d} t
$$

it follows from (22) and (23) that

$$
\left|\int_{-\infty}^{\infty} f(\tau) h_{\delta}(\tau) \mathrm{d} \tau\right| \leq 3\left\|f_{\omega_{1}}\right\|_{\infty}
$$

for all $f \in \mathcal{B}_{\pi, 0}^{\infty}$ and all $0<\delta<\omega_{1}$.

Next, consider the functionals $\Phi_{\delta} f: \mathcal{B}_{\pi, 0}^{\infty} \rightarrow \mathbb{C}, 0<\delta<\omega_{1}$, given by

$$
\Phi_{\delta} f=\int_{-\infty}^{\infty} f(\tau) h_{\delta}(\tau) \mathrm{d} \tau
$$

Clearly, for all $0<\delta<\omega_{1}, \Phi_{\delta}$ is a bounded linear functional. Since

$$
\sup _{0<\delta<\omega_{1}}\left|\Phi_{\delta} f\right| \leq 3\left\|f_{\omega_{1}}\right\|_{\infty}<\infty
$$

for all $f \in \mathcal{B}_{\pi, 0}^{\infty}$, it follows by the Banach-Steinhaus theorem $\left[13\right.$, p. 98] that there exists a constant $C_{3}<\infty$ such that

$$
\sup _{0<\delta<\omega_{1}} \sup _{\substack{f \in \mathcal{B}_{\pi, 0}^{\infty},\|f\|_{\mathcal{B}_{\pi, 0}^{\infty} \leq 1}^{\infty} \leq 1}}\left|\Phi_{\delta} f\right| \leq C_{3} .
$$


Moreover, the limit functional $\Phi f=\lim _{\delta \rightarrow 0} \Phi_{\delta} f$ is a well defined bounded linear functional on $\mathcal{P} \mathcal{W}_{\pi}^{2}$, because for all $f \in$ $\mathcal{P} \mathcal{W}_{\pi}^{2}$ we have

$$
\Phi f=\lim _{\delta \rightarrow 0} \Phi_{\delta} f=\int_{-\infty}^{\infty} f(\tau) \frac{\sin \left(\omega_{1} \tau\right)}{\pi \tau} \mathrm{d} \tau .
$$

Since $\mathcal{P} \mathcal{W}_{\pi}^{2}$ is dense in $\mathcal{B}_{\pi, 0}^{\infty}$, it follows that the limit $\Phi f=$ $\lim _{\delta \rightarrow 0} \Phi_{\delta} f$ exists also for all $f \in \mathcal{B}_{\pi, 0}^{\infty}$ and that there exists a constant $C_{4}<\infty$ such that

$$
\sup _{\substack{f \in \mathcal{B}_{\pi, 0}^{\infty} \\\|f\|_{\mathcal{B}}^{\infty} \leq, 0}}|\Phi f| \leq C_{4} .
$$

Since $\mathcal{P} \mathcal{W}_{\pi}^{2} \subset \mathcal{B}_{\pi, 0}^{\infty}$, we further obtain

$$
\begin{aligned}
\sup _{\substack{f \in \mathcal{P} \mathcal{W}_{\pi}^{2},\|f\|_{\mathcal{B}}^{\infty}, 01}}\left|\int_{-\infty}^{\infty} f(\tau) \frac{\sin \left(\omega_{1} \tau\right)}{\pi \tau} \mathrm{d} \tau\right| & =\sup _{\substack{f \in \mathcal{P} \mathcal{W}_{\pi}^{2} \\
\|f\|_{\mathcal{B}^{\infty}}^{\infty} \leq 1}}|\Phi f| \\
& \leq \sup _{\substack{f \in \mathcal{B}^{\infty}, 0 \\
\|f\|_{\mathcal{B}^{\infty}, 0}^{\infty} \leq 1}}^{\infty}|\Phi f| \\
& \leq C_{4} .
\end{aligned}
$$

Let $C_{5}=\min \left\{\pi-\omega_{1}, \omega_{1}\right\}$. For $N \in \mathbb{N}$, consider the functions

$$
f_{N}(t)=\frac{\mathrm{e}^{i \omega_{1} t}}{i} \frac{\sin \left(\frac{t}{2 N}\right)}{\frac{t}{2 N}} \sum_{k=1}^{\left\lfloor N \gamma_{N}\right\rfloor} \frac{1}{k} \sin \left(\frac{k t}{N}\right)
$$

where $\gamma_{N}=C_{5}-1 /(2 N)$. Since

$$
\left|f_{N}(t)\right| \leq\left|\sum_{k=1}^{\left\lfloor N \gamma_{N}\right\rfloor} \frac{1}{k} \sin \left(\frac{k t}{N}\right)\right|
$$

and

$$
\left\|\sum_{k=1}^{\left\lfloor N \gamma_{N}\right\rfloor} \frac{1}{k} \sin \left(\frac{k \cdot}{N}\right)\right\|_{\infty} \leq C_{1}
$$

according to (8), where the constant $C_{1}$ is independent of $N$, we have

$$
\left\|f_{N}\right\|_{\infty} \leq C_{1}<\infty
$$

for all $N \in \mathbb{N}$.

Using (25), we obtain

$$
\begin{aligned}
\int_{-\infty}^{\infty}\left|f_{N}(t)\right|^{2} \mathrm{~d} t & =\int_{-\infty}^{\infty}\left(\frac{\sin \left(\frac{t}{2 N}\right)}{\frac{t}{2 N}}\right)^{2}\left|\sum_{k=1}^{\left\lfloor N \gamma_{N}\right\rfloor} \frac{1}{k} \sin \left(\frac{k t}{N}\right)\right|^{2} \mathrm{~d} t \\
& \leq C_{1}^{2} \int_{-\infty}^{\infty}\left(\frac{\sin \left(\frac{t}{2 N}\right)}{\frac{t}{2 N}}\right)^{2} \mathrm{~d} t \\
& =2 C_{1}^{2} N \pi
\end{aligned}
$$

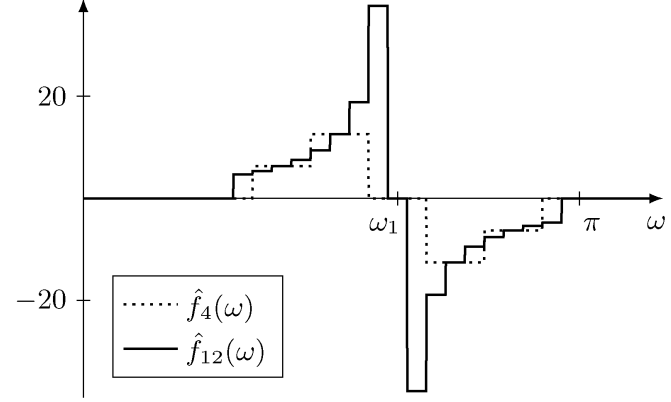

Fig. 4. Plot of $\hat{f}_{N}(\omega)$ for $N=4$ (dotted line) and $N=12$ (solid line).

which shows that $f_{N} \in L^{2}(\mathbb{R})$ for all $N \in \mathbb{N}$. Moreover, since the Fourier transform of $f_{N}$ is given by

$$
\begin{aligned}
\hat{f}_{N}(\omega)=\sum_{k=1}^{\left\lfloor N \gamma_{N}\right\rfloor} \frac{N \pi}{k}\left[1_{\left[-\frac{1}{2 N}, \frac{1}{2 N}\right]}\left(\omega-\omega_{1}+\frac{k}{N}\right)\right. \\
\left.-1_{\left[-\frac{1}{2 N}, \frac{1}{2 N}\right]}\left(\omega-\omega_{1}-\frac{k}{N}\right)\right] .
\end{aligned}
$$

we see that all $f_{N}$ are bandlimited with bandwidth $\pi . \hat{f}_{N}$ is depicted in Fig. 4 for $N=4$ and $N=12$. Thus, $f_{N} \in \mathcal{P} \mathcal{W}_{\pi}^{2}$ for all $N \in \mathbb{N}$. It follows that the functions $g_{N}=f_{N} / C_{1}, N \in \mathbb{N}$, are all in $\mathcal{P W}_{\pi}^{2}$ and have a norm $\left\|g_{N}\right\|_{\mathcal{B}_{\pi, 0}^{\infty}} \leq 1$. According to Parseval's theorem, we have

$$
\int_{-\infty}^{\infty} f_{N}(\tau) \frac{\sin \left(\omega_{1} \tau\right)}{\pi \tau} \mathrm{d} \tau=\frac{1}{2 \pi} \int_{-\omega_{1}}^{\omega_{1}} \hat{f}_{N}(\omega) \mathrm{d} \omega
$$

and it follows that

$$
\begin{aligned}
& \int_{-\infty}^{\infty} f_{N}(t) \frac{\sin \left(\omega_{1} t\right)}{\pi t} \mathrm{~d} t \\
& =\sum_{k=1}^{\left\lfloor N \gamma_{N}\right\rfloor} \frac{N}{2 k} \int_{-\omega_{1}}^{\omega_{1}} \mathbf{1}_{\left[-\frac{1}{2 N}, \frac{1}{2 N}\right]}\left(\omega-\omega_{1}+\frac{k}{N}\right) \mathrm{d} \omega \\
& =\frac{1}{2} \sum_{k=1}^{\left\lfloor N \gamma_{N}\right\rfloor} \frac{1}{k} \\
& \geq \frac{1}{2} \log \left(N \gamma_{N}\right) \\
& =\frac{1}{2} \log \left(N C_{5}-\frac{1}{2}\right)
\end{aligned}
$$

for all $N \in \mathbb{N}, N>1 /\left(2 C_{5}\right)$. Consequently, we have

$$
\int_{-\infty}^{\infty} g_{N}(t) \frac{\sin \left(\omega_{1} t\right)}{\pi t} \mathrm{~d} t \geq \frac{1}{2 C_{1}} \log \left(N C_{5}-\frac{1}{2}\right)
$$

for all $N \in \mathbb{N}, N>1 /\left(2 C_{5}\right)$. Furthermore, since

$$
\sup _{\substack{f \in \mathcal{P} \mathcal{W},\|f\|_{\mathcal{B}}^{\infty}, \pi \\ \pi, 0}}\left|\int_{-\infty}^{\infty} f(\tau) \frac{\sin \left(\omega_{1} \tau\right)}{\pi \tau} \mathrm{d} \tau\right| \geq \int_{-\infty}^{\infty} g_{N}(\tau) \frac{\sin \left(\omega_{1} \tau\right)}{\pi \tau} \mathrm{d} \tau
$$




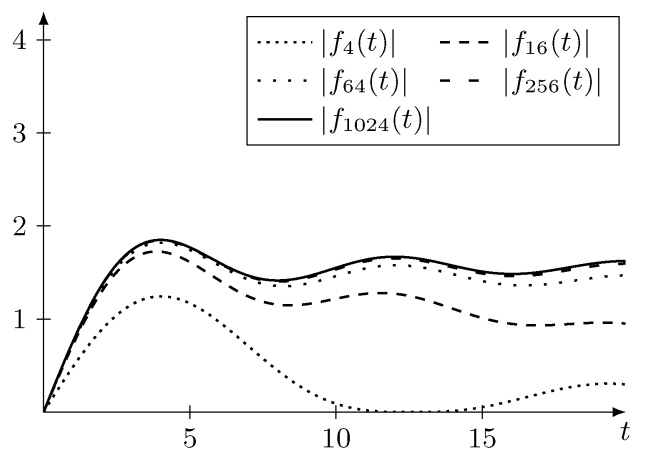

Fig. 5. Plot of $\left|f_{N}(t)\right|$ for $N=4,16,64,256,1024$, illustrating that $\left\|f_{N}\right\|_{\infty} \leq C_{1}$.

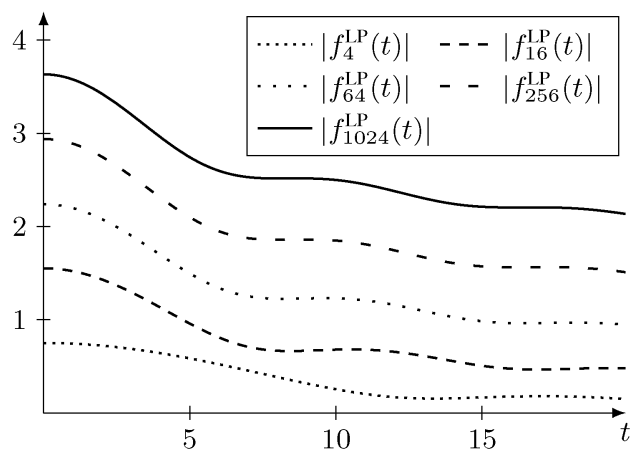

Fig. 6. Plot of $\left|f_{N}^{\mathrm{LP}}(t)\right|$ for $N=4,16,64,256,1024$, illustrating the unbounded increase of $\left\|f_{N}^{\mathrm{LP}}\right\|_{\infty}$.

we obtain

$$
\sup _{\substack{f \in \mathcal{P} \mathcal{W}_{\pi}^{2},\|f\|_{\mathcal{B}}, 0}}\left|\int_{-\infty}^{\infty} f(\tau) \frac{\sin \left(\omega_{1} \tau\right)}{\pi \tau} \mathrm{d} \tau\right| \geq \frac{1}{2 C_{1}} \log \left(N C_{5}-\frac{1}{2}\right)
$$

for all $N \in \mathbb{N}, N>1 /\left(2 C_{5}\right)$, and it follows that

$$
\sup _{\substack{f \in \mathcal{P} \mathcal{W}_{\pi}^{2},\|f\|_{\mathcal{B}}^{\infty} \leq 1}}\left|\int_{-\infty}^{\infty} f(\tau) \frac{\sin \left(\omega_{1} \tau\right)}{\pi \tau} \mathrm{d} \tau\right|=\infty .
$$

This is a contradiction to (24).

\section{A. Illustration of the No-Go Theorem for Frequency Splitting}

Before we proceed in the discussion, we illustrate Theorem 3 with two plots. To obtain them, we have chosen $\omega_{1}=3 \pi / 4$. In Fig. 5 we see the absolute values of the signals $f_{N}$, which were used in the proof of Theorem 3 , for $N=4,16,64,256,1024$. It is clearly visible that the $L^{\infty}$-norm of the signals does not grow infinitely as $N$ is increased. In fact, the $L^{\infty}$-norm of all signals is bounded above by the same constant (the constant $C_{1}$ in the proof of Theorem 3).

Fig. 6 shows the absolute values of the signals $f_{N}^{\mathrm{LP}}$ that are obtained by a lowpass filtering of the signals $f_{N}$ according to

$$
f_{N}^{\mathrm{LP}}(t)=\int_{-\infty}^{\infty} f_{N}(\tau) \frac{\sin \left(\omega_{1}(t-\tau)\right)}{\pi(t-\tau)} \mathrm{d} \tau .
$$

The unbounded increase of $\left\|f_{N}^{\mathrm{LP}}\right\|_{\infty}$ as $N$ is increased is visible.

\section{B. Approximate Frequency Splitting}

From Theorem 3, we have seen that a frequency splitting is not possible for signals in the space $\mathcal{B}_{\pi, 0}^{\infty}$ in general. Of course, an exact frequency splitting, as discussed in Section VI, is a high requirement and not always necessary for applications. That is why we want to analyze in this section whether an approximate frequency splitting is possible and how the peak value of the signal behaves as the approximate splitting approaches more and more the exact splitting.

For $0<\omega_{1}<\infty, \delta>0$, and $1<\kappa<\infty$ let $\overline{\mathcal{K}}\left(\omega_{1}, \delta, \kappa\right)$ denote the set of all functions $K \in L^{1}(\mathbb{R})$, whose Fourier transform fulfills $\|\hat{K}\|_{\infty} \leq \kappa, \hat{K}(\omega)=1$ for $|\omega| \leq \omega_{1}$, and $\hat{K}(\omega)=0$ for $|\omega|>\omega_{1}+\delta$. For $K \in \overline{\mathcal{K}}\left(\omega_{1}, \delta, \kappa\right)$ we further define the system $\Psi_{K}: \mathcal{B}_{\pi, 0}^{\infty} \rightarrow \mathcal{B}_{\omega_{1}+\delta, 0}^{\infty}$ by

$$
\left(\Psi_{K} f\right)(t)=\int_{-\infty}^{\infty} f(\tau) K(t-\tau) \mathrm{d} \tau
$$

For input signals in $\mathcal{P} \mathcal{W}_{\pi}^{2}, \hat{K}$ has the meaning of a transfer function, and we see that the definition of $K \in \overline{\mathcal{K}}\left(\omega_{1}, \delta, \kappa\right)$ is a relaxation of P1). We only require that $\hat{K}(\omega)=0$ for $|\omega|>\omega_{1}+\delta$, and apart from the boundedness of $\hat{K}$ we make no assumptions about the shape of $\hat{K}$ in the transition region $\left(-\omega_{1}-\delta,-\omega_{1}\right) \cup\left(\omega_{1}, \omega_{1}+\delta\right)$.

It follows from Lemma 5 that $\Psi_{K}$ is a $\mathrm{BIBO}_{\pi}$-stable LTI system. Thus, an approximate frequency splitting is possible for $\mathcal{B}_{\pi}^{\infty}$. However, the following theorem shows that peak value of the output signal $\left\|\Psi_{K} f\right\|_{\infty}$ increases unboundedly as the width of the transition region $\delta$ is reduced, independently of the chosen $K \in \overline{\mathcal{K}}\left(\omega_{1}, \delta, \kappa\right)$.

Theorem 4: Let $0<\omega_{1}<\pi$ and $1<\kappa<\infty$. Then there exists a function $q_{\omega_{1}, \kappa}: \mathbb{R}^{+} \rightarrow \mathbb{R}^{+}$with

$$
\lim _{\delta \rightarrow 0} q_{\omega_{1}, \kappa}(\delta)=\infty
$$

such that for all $\delta>0$ we have

$$
\left\|\Psi_{K}\right\|_{B I B O_{\pi}} \geq q_{\omega_{1}, \kappa}(\delta)
$$

for all $K \in \overline{\mathcal{K}}\left(\omega_{1}, \delta, \kappa\right)$.

Remark 5: An equivalent way to formulate the assertion of Theorem 4 is the following. For all $0<\omega_{1}<\pi$ and $1<\kappa<\infty$ we have

$$
\liminf _{\delta \rightarrow 0} \inf _{K \in \overline{\mathcal{K}}\left(\omega_{1}, \delta, \kappa\right)}\left\|\Psi_{K}\right\|_{\mathrm{BIBO}_{\pi}}=\infty .
$$

The proof of Theorem 4 is a simple application of the ideas that were developed so far.

Proof of Theorem 4: Let $0<\omega_{1}<\pi$ and $1<\kappa<\infty$ be arbitrary but fixed. We assume that the negation of the assertion is true and construct a contradiction. Thus, we assume that there exist a constant $C_{6}<\infty$ and a sequence $\left\{\delta_{n}\right\}_{n \in \mathbb{N}}$ converging to zero such that for every $n \in \mathbb{N}$ there exists a $K_{n} \in \overline{\mathcal{K}}\left(\omega_{1}, \delta_{n}, \kappa\right)$ with

$$
\left\|\Psi_{K_{n}}\right\|_{\mathrm{BIBO}_{\pi}} \leq C_{6}
$$


Let $t=0$. For $f \in \mathcal{P} \mathcal{W}_{\pi}^{2}$ consider the functionals

$$
\Phi_{n} f:=\left(\Psi_{K_{n}} f\right)(0)=\frac{1}{2 \pi} \int_{-\omega_{1}-\delta}^{\omega_{1}+\delta} \hat{f}(\omega) \hat{K}_{n}(\omega) \mathrm{d} \omega .
$$

Clearly, for all $n \in \mathbb{N}, \Phi_{n}$ is a bounded linear functional. Furthermore, since $\left\|\hat{K}_{n}\right\|_{\infty} \leq \kappa$ for all $n \in \mathbb{N}$ and

$$
\lim _{n \rightarrow \infty} \hat{K}_{n}(\omega)=\mathbf{1}_{\left[-\omega_{1}, \omega_{1}\right]}(\omega)
$$

where

$$
1_{\left[-\omega_{1}, \omega_{1}\right]}(\omega)= \begin{cases}1, & \omega \in\left[-\omega_{1}, \omega_{1}\right] \\ 0, & \text { otherwise }\end{cases}
$$

denotes the indicator function, we can apply Lebesgue's dominated convergence theorem to obtain

$$
\lim _{n \rightarrow \infty} \Phi_{K_{n}} f=\frac{1}{2 \pi} \int_{-\omega_{1}}^{\omega_{1}} \hat{f}(\omega) \mathrm{d} \omega=\int_{-\infty}^{\infty} f(\tau) \frac{\sin \left(\omega_{1} \tau\right)}{\pi \tau} \mathrm{d} \tau .
$$

Thus, the limit functional $\Phi f=\lim _{n \rightarrow \infty} \Phi_{n} f$ is a well defined bounded linear functional on $\mathcal{P} \mathcal{W}_{\pi}^{2}$. Since $\mathcal{P} \mathcal{W}_{\pi}^{2}$ is dense in $\mathcal{B}_{\pi, 0}^{\infty}$, it follows the limit $\Phi f=\lim _{n \rightarrow \infty} \Phi_{n} f$ exists also for all $f \in \mathcal{B}_{\pi, 0}^{\infty}$, and that there exists a constant $C_{7}<\infty$ such that

$$
\sup _{\substack{f \in \mathcal{B}_{\pi, 0}^{\infty} \\\|f\|_{\mathcal{B}_{\pi, 0}^{\infty} \leq 1}^{\infty} \leq}}|\Phi f| \leq C_{7}
$$

Since $\mathcal{P} \mathcal{W}_{\pi}^{2} \subset \mathcal{B}_{\pi, 0}^{\infty}$, we further obtain

$$
\begin{aligned}
\sup _{\substack{f \in \mathcal{P} \mathcal{W}_{\pi}^{2} \\
\|f\|_{\mathcal{B}_{\pi, 0}^{\infty} \leq 1}^{\infty}}}\left|\int_{-\infty}^{\infty} f(\tau) \frac{\sin \left(\omega_{1} \tau\right)}{\pi \tau} \mathrm{d} \tau\right| & =\sup _{\substack{f \in \mathcal{P} \mathcal{W}_{\pi}^{2} \\
\|f\|_{\mathcal{B}_{\pi, 0}^{\infty} \leq 1}^{\infty}}}|\Phi f| \\
& \leq \sup _{\substack{f \in \mathcal{B}_{\pi, 0}^{\infty} \\
\|f\|_{\mathcal{B}_{\pi, 0}^{\infty} \leq 1}^{\infty}}}|\Phi f| \leq C_{7} .
\end{aligned}
$$

This is a contradiction to the fact

$$
\sup _{\substack{f \in \mathcal{P} \mathcal{W}_{\pi}^{2},\|f\|_{\mathcal{B}}^{\infty} \leq 1}}\left|\int_{-\infty}^{\infty} f(\tau) \frac{\sin \left(\omega_{1} \tau\right)}{\pi \tau} \mathrm{d} \tau\right|=\infty
$$

which was derived in the proof of Theorem 3.

\section{Further Discussion of the No-Go Theorem for Frequency Splitting}

Theorem 3 states the existence of a signal in $\mathcal{B}_{\pi, 0}^{\infty}$, for which a frequency splitting is not possible. The following corollary shows that this phenomenon is no singular event for a single signal. In a precise sense "almost all" signals in $\mathcal{B}_{\pi, 0}^{\infty}$ have the property that a frequency splitting is not possible.

In order to specify what we mean with "almost all" we need the following concepts from normed spaces. A subset $M$ of a normed space $X$ is said to be nowhere dense in $X$ if the closure $[M]$ does not contain a nonempty open set of $X . M$ is said to be of the first category (or meager) if $M$ is the countable union of sets each of which is nowhere dense in $X . M$ is said to be of the second category (or nonmeager) if it is not of the first category. The complement of a set of the first category is called a residual set. Sets of first category may be considered as "small". According to Baire's theorem [14] we have that in a complete normed space, the residual set is dense and a set of the second category. One property that shows the abundance of residual sets is the following: the countable intersection of residual sets is always a residual set.

Corollary 1: Let $0<\omega_{1}<\pi$. The set of signals $f \in \mathcal{B}_{\pi, 0}^{\infty}$ for which there exists no signal $f_{\omega_{1}} \in \mathcal{B}_{\omega_{1}}^{\infty}$ such that (21) is true for all $h \in \mathcal{B}_{\omega_{1}}^{1}$ is a residual set.

Proof: Let $0<\omega_{1}<\pi$ be arbitrary but fixed, and let $\mathcal{F S}_{\omega_{1}}$ denote the set of all signals in $\mathcal{B}_{\pi, 0}^{\infty}$ for which there exists a signal $f_{\omega_{1}} \in \mathcal{B}_{\omega_{1}}^{\infty}$ such that (21) is true for all $h \in \mathcal{B}_{\omega_{1}}^{1}$. Further, let

$$
\hat{K}_{\delta}(\omega)= \begin{cases}1, & |w| \leq \omega_{1} \\ 1-\frac{|\omega|-\omega_{1}}{\delta} & \omega_{1}<|\omega|<\omega_{1}+\delta, \\ 0 & |\omega| \geq \omega_{1}+\delta\end{cases}
$$

and $\left\{\delta_{n}\right\}_{n \in \mathbb{N}}$ a sequence of positive numbers converging to zero.

Let $f \in \mathcal{F S}_{\omega_{1}}$. We have

$$
\begin{aligned}
\left(T_{n} f\right)(t) & :=\int_{-\infty}^{\infty} f(\tau) K_{\delta_{n}}(t-\tau) \mathrm{d} \tau \\
& =\int_{-\infty}^{\infty} f(\tau)\left(h_{\delta_{n}}(t-\tau)+r_{\delta_{n}}(t-\tau)\right) \mathrm{d} \tau
\end{aligned}
$$

where $h_{\delta}$ and $r_{\delta}$ are the functions that were used in the proof of Theorem 3, and whose Fourier transforms are depicted in Fig. 3. Moreover, since $f \in \mathcal{F} \mathcal{S}_{\omega_{1}}$, we have

$$
\begin{aligned}
\int_{-\infty}^{\infty} f(\tau) h_{\delta_{n}}(t-\tau) \mathrm{d} \tau & =\int_{-\infty}^{\infty} f_{\omega_{1}}(\tau) h_{\delta_{n}}(t-\tau) \mathrm{d} \tau \\
& \leq 3\left\|f_{\omega_{1}}\right\|_{\infty}
\end{aligned}
$$

where we used the same steps as in (23) to obtain the last inequality. Furthermore

$$
\int_{-\infty}^{\infty} f(\tau) r_{\delta_{n}}(t-\tau) \leq\|f\|_{\infty} \int_{-\infty}^{\infty}\left|r_{\delta_{n}}(\tau)\right| \mathrm{d} \tau \leq 2\|f\|_{\infty}
$$

according to (22). Combining (26), (27), and (28), we obtain $\left(T_{n} f\right)(t) \leq 3\left\|f_{\omega_{1}}\right\|_{\infty}+2\|f\|_{\infty}$ for all $f \in \mathcal{F S}_{\omega_{1}}$ and all $t \in \mathbb{R}$, and consequently $\lim \sup _{n \rightarrow \infty}\left\|T_{n} f\right\|_{\infty}<\infty$ for all $f \in \mathcal{F S}_{\omega_{1}}$.

Let $\mathcal{D}_{\omega_{1}}$ denote the set of signals in $\mathcal{B}_{\pi, 0}^{\infty}$ for which $\limsup _{n \rightarrow \infty}\left\|T_{n} f\right\|_{\infty}=\infty$. We have $\mathcal{F} \mathcal{S}_{\omega_{1}} \subseteq \mathcal{B}_{\pi, 0}^{\infty} \backslash \mathcal{D}_{\omega_{1}}$. According to Theorem 4 we have

$$
\limsup _{n \rightarrow \infty} \sup _{\substack{f \in \mathcal{B}_{\pi, 0}^{\infty} \\\|f\|_{\mathcal{B}}^{\infty}, 0}}\left\|T_{n} f\right\|_{\infty}=\infty .
$$

Hence, the Banach-Steinhaus theorem [15, p. 251] implies that $\mathcal{D}_{\omega_{1}}$ is a residual set. Thus, by definition, $\mathcal{B}_{\pi, 0}^{\infty} \backslash \mathcal{D}_{\omega_{1}}$ is a set of first category. Since $\mathcal{F} \mathcal{S}_{\omega_{1}}$ is a subset of $\mathcal{B}_{\pi, 0}^{\infty} \backslash \mathcal{D}_{\omega_{1}}$, it is 
also a set of first category. Consequently, the set $\mathcal{B}_{\pi, 0}^{\infty} \backslash \mathcal{F} \mathcal{S}_{\omega_{1}}$, i.e., the set of signals $f \in \mathcal{B}_{\pi, 0}^{\infty}$ for which there exists no signal $f_{\omega_{1}} \in \mathcal{B}_{\omega_{1}}^{\infty}$ such that (21) is true for all $h \in \mathcal{B}_{\omega_{1}}^{1}$, is a residual set.

\section{DISCUSSION AND CONCLUDING REMARKS}

We have analyzed the existence and realizability of bandpass-type systems for the space $\mathcal{B}_{\pi}^{\infty}$. It has been shown that a linear realization of efficient bandpass-type systems is impossible, because the corresponding operator does not exist. Moreover, we have shown in Theorem 2 that a nonlinear realization of efficient bandpass-type systems is possible. In practice, nonlinear systems are in general more difficult to implement than linear system. Thus, we assume that there is no simple realization of the nonlinear operator. The proof of Theorem 2 rather suggests that the realization of a system with the properties P1)-P3) requires complex algorithms.

In the paper, we considered systems that fulfill property P1), i.e., systems whose output signals agree with the zero function outside the passband. As already indicated in the introduction, this requirement is relatively high and reflects the ideal situation in many applications. However, certain applications allow the output signals to have a small portion of the signal outside the passband. For example, in mobile communications the transmitted signal has only to fulfill a spectral mask, which specifies that the energy spectral density of the signal has to be below some threshold. Thus, it would be interesting to extend the ideas in this paper toward a more relaxed version of property P1).

A further result of this paper is that a frequency splitting, which is conceptually related to the existence of ideal bandpasstype systems, is not possible for signals in $\mathcal{B}_{\pi}^{\infty}$ in general. This shows that the common way of thinking, which suggests that a signal can be divided according to its frequencies, is not justified for the space $\mathcal{B}_{\pi}^{\infty}$.

\section{APPENDIX}

\section{A. Proof of Lemma 2}

Proof of Lemma 2: Since a closed subspace of a complete normed space is complete, it suffices to show that $\mathcal{B}_{\pi, 0}^{\infty}$ is closed in $\mathcal{B}_{\pi}^{\infty}$. Let $\left\{f_{k}\right\}_{k \in \mathbb{N}} \subset \mathcal{B}_{\pi, 0}^{\infty}$ be a sequence that converges to some $f \in \mathcal{B}_{\pi}^{\infty}$. we have to show that $f \in \mathcal{B}_{\pi, 0}^{\infty}$, i.e., that $\lim _{t \rightarrow \infty} f(t)=0$. Let $\epsilon>0$ be arbitrary but fixed. By assumption, there exists a $k_{0} \in \mathbb{N}$ such that $\left\|f_{k}-f\right\|_{\infty}<\epsilon / 2$ for all $k \geq k_{0}$. Now fix $k_{0}$. Since $f_{k_{0}} \in \mathcal{B}_{\pi, 0}^{\infty}$, there exists a $t_{0} \in \mathbb{R}$ such that $\left|f_{k_{0}}(t)\right|<\epsilon / 2$ for all $|t|>t_{0}$. Thus, it follows that

$$
\begin{aligned}
|f(t)| & \leq\left|f_{k_{0}}(t)-f(t)\right|+\left|f_{k_{0}}(t)\right| \\
& \leq\left\|f_{k_{0}}-f\right\|_{\infty}+\left|f_{k_{0}}(t)\right|<\epsilon
\end{aligned}
$$

for all $|t| \geq t_{0}$.

\section{B. Proof of Lemma 3}

Proof of Lemma 3: Let $\sigma>0$ be arbitrary but fixed.

First we show that $f \in \mathcal{B}_{+[-\sigma, \sigma]}^{\infty}$ implies $f \in \mathcal{B}_{\sigma}^{\infty}$. Let $f \in$ $\mathcal{B}_{+[-\sigma, \sigma]}^{\infty}$. For $\epsilon>0$, arbitrary but fixed, choose some $K_{\epsilon} \in$
$\mathcal{K}_{+}(-\sigma, \sigma) \cap \mathcal{B}_{\sigma+\epsilon}^{1}$. According to the definition of the space $\mathcal{B}_{+[-\sigma, \sigma]}^{\infty}$ we have

$$
f(t)=\int_{-\infty}^{\infty} f(\tau) K_{\epsilon}(t-\tau) \mathrm{d} \tau, \quad t \in \mathbb{R} .
$$

It follows that

$$
f^{(k)}(0)=\int_{-\infty}^{\infty} f(\tau) K_{\epsilon}^{(k)}(-\tau) \mathrm{d} \tau .
$$

Since $\left\|K_{\epsilon}^{(k)}\right\|_{1} \leq(\sigma+\epsilon)^{k}\left\|K_{\epsilon}\right\|_{1}$, due to Bernstein's Inequality $[9$, p. 49], we obtain

$$
\left|f^{(k)}(0)\right| \leq\|f\|_{\infty}\left\|K_{\epsilon}^{(k)}\right\|_{1} \leq\|f\|_{\infty}(\sigma+\epsilon)^{k}\left\|K_{\epsilon}\right\|_{1} .
$$

Thus, the Taylor series expansion of $f$ is given by

$$
f(z)=\sum_{k=0}^{\infty} \frac{f^{(k)}(0)}{k !} z^{k} .
$$

It follows that

$|f(z)| \leq\|f\|_{\infty}\left\|K_{\epsilon}\right\|_{1} \sum_{k=0}^{\infty} \frac{(\sigma+\epsilon)^{k}}{k !}|z|^{k}=\|f\|_{\infty}\left\|K_{\epsilon}\right\|_{1} \mathrm{e}^{(\sigma+\epsilon)|z|}$

which holds for all $\epsilon>0$, because $\epsilon>0$ was arbitrary. This shows that $f \in \mathcal{B}_{\sigma}^{\infty}$.

Next, we show that $f \in \mathcal{B}_{\sigma}^{\infty}$ implies $f \in \mathcal{B}_{+[-\sigma, \sigma]}^{\infty}$. Let $f \in \mathcal{B}_{\sigma}^{\infty}$. We have to show that

$$
f(t)=\int_{-\infty}^{\infty} f(\tau) K(t-\tau) \mathrm{d} \tau
$$

for all $t \in \mathbb{R}$ and all $K \in \mathcal{K}_{+}(-\sigma, \sigma)$. Let $t \in \mathbb{R}$ and $K \in$ $\mathcal{K}_{+}(-\sigma, \sigma)$ be arbitrary but fixed. For $0<\epsilon<1$ consider the family of functions

$$
f_{\epsilon}(t)=f((1-\epsilon) t) \frac{\sin (\epsilon \pi t)}{\epsilon \pi t} .
$$

We have

$$
\lim _{\epsilon \rightarrow 0} f_{\epsilon}(t)=f(t) .
$$

Moreover, since $f_{\epsilon} \in \mathcal{P} \mathcal{W}_{\sigma}^{2}$, we have the identity

$$
\begin{aligned}
\int_{-\infty}^{\infty} f_{\epsilon}(\tau) K(t-\tau) \mathrm{d} \tau & =\frac{1}{2 \pi} \int_{-\sigma}^{\sigma} \hat{f}_{\epsilon}(\omega) \hat{K}(\omega) \mathrm{e}^{i \omega t} \mathrm{~d} \omega \\
& =\frac{1}{2 \pi} \int_{-\sigma}^{\sigma} \hat{f}_{\epsilon}(\omega) \mathrm{e}^{i \omega t} \mathrm{~d} \omega \\
& =f_{\epsilon}(t)
\end{aligned}
$$

for all $0<\epsilon<1$, and consequently

$$
\lim _{\epsilon \rightarrow 0} \int_{-\infty}^{\infty} f_{\epsilon}(\tau) K(t-\tau) \mathrm{d} \tau=f(t) .
$$


Further, according to Lebesgue's dominated convergence theorem, which can be applied because

$$
\left|f_{\epsilon}(\tau) K(t-\tau)\right| \leq\left\|f_{\epsilon}\right\|_{\infty}|K(t-\tau)|
$$

and $K \in L^{1}(\mathbb{R})$, we have

$$
\lim _{\epsilon \rightarrow 0} \int_{-\infty}^{\infty} f_{\epsilon}(\tau) K(t-\tau) \mathrm{d} \tau=\int_{-\infty}^{\infty} f(\tau) K(t-\tau) \mathrm{d} \tau .
$$

Combining (30) and (31) shows that

$$
f(t)=\int_{-\infty}^{\infty} f(\tau) K(t-\tau) \mathrm{d} \tau
$$

which completes the proof, because $t \in \mathbb{R}$ and $K \in \mathcal{K}_{+}(-\sigma, \sigma)$ were arbitrary.

\section{Proof of Lemma 4}

Proof of Lemma 4: Let $-\infty<\omega_{1}<\omega_{2}<\infty$ be arbitrary but fixed and $\sigma=\max \left\{\left|\omega_{1}\right|,\left|\omega_{2}\right|\right\}$. First, we show that $\mathcal{B}_{+\left[\omega_{1}, \omega_{2}\right]}^{\infty}$ is a subspace of $\mathcal{B}_{\sigma}^{\infty}$. Since $\mathcal{B}_{+\left[\omega_{1}, \omega_{2}\right]}^{\infty}$ is obviously a linear space, it remains to show that $f \in \mathcal{B}_{+\left[\omega_{1}, \omega_{2}\right]}^{\infty}$ implies $f \in \mathcal{B}_{\sigma}^{\infty}$. Let $f \in \mathcal{B}_{+\left[\omega_{1}, \omega_{2}\right]}^{\infty}$. Then we have

$$
f(t)=\int_{-\infty}^{\infty} f(\tau) K(t-\tau) \mathrm{d} \tau
$$

for all $t \in \mathbb{R}$ and $K \in \mathcal{K}_{+}\left(\omega_{1}, \omega_{2}\right)$. Since $\mathcal{K}_{+}(-\sigma, \sigma) \subset$ $\mathcal{K}_{+}\left(\omega_{1}, \omega_{2}\right),(32)$ is also true for all $t \in \mathbb{R}$ and all $\mathcal{K}_{+}(-\sigma, \sigma)$. Thus, $f \in \mathcal{B}_{+[-\sigma, \sigma]}$. This completes the first part of the proof, because $\mathcal{B}_{+[-\sigma, \sigma]}=\mathcal{B}_{\sigma}^{\infty}$, according to Lemma 3 .

Next, we show that $\mathcal{B}_{+\left[\omega_{1}, \omega_{2}\right]}^{\infty}$ is closed and complete. Let $\left\{f_{k}\right\}_{k \in \mathbb{N}}$ be a Cauchy sequence of functions in $\mathcal{B}_{+\left[\omega_{1}, \omega_{2}\right]}^{\infty}$. Thus, $\left\{f_{k}\right\}_{k \in \mathbb{N}}$ is also a Cauchy sequence in $\mathcal{B}_{\sigma}^{\infty}$, and because $\mathcal{B}_{\sigma}^{\infty}$ is complete, there exists a function $f \in \mathcal{B}_{\sigma}^{\infty}$ such that

$$
\lim _{k \rightarrow \infty}\left\|f-f_{k}\right\|_{\infty}=0 .
$$

We have to show that $f \in \mathcal{B}_{+\left[\omega_{1}, \omega_{2}\right]}^{\infty}$. Let $t \in \mathbb{R}$ and $K \in$ $\mathcal{K}_{+}\left(\omega_{1}, \omega_{2}\right)$ be arbitrary but fixed. We have

$$
\begin{aligned}
& \left|f(t)-\int_{-\infty}^{\infty} f(\tau) K(t-\tau) \mathrm{d} \tau\right| \\
& =\left|f(t)-f_{k}(t)+\int_{-\infty}^{\infty}\left(f_{k}(\tau)-f(\tau)\right) K(t-\tau) \mathrm{d} \tau\right| \\
& \left.\quad \leq\left|f(t)-f_{k}(t)\right|+\int_{-\infty}^{\infty} \mid f(\tau)-f_{k}(\tau)\right)|| K(t-\tau) \mid \mathrm{d} \tau \\
& \quad \leq|| f-f_{k} \|_{\infty}\left(1+\int_{-\infty}^{\infty}|K(\tau)| \mathrm{d} \tau\right) .
\end{aligned}
$$

Taking the limit $k \rightarrow \infty$ and using (33) gives

$$
\left|f(t)-\int_{-\infty}^{\infty} f(\tau) K(t-\tau) \mathrm{d} \tau\right|=0 .
$$

Since $t \in \mathbb{R}$ and $K \in \mathcal{K}_{+}\left(\omega_{1}, \omega_{2}\right)$ were chosen arbitrary, we have

$$
f(t)=\int_{-\infty}^{\infty} f(\tau) K(t-\tau)
$$

for all $t \in \mathbb{R}$ and $K \in \mathcal{K}_{+}\left(\omega_{1}, \omega_{2}\right)$. This shows that $f \in$ $\mathcal{B}_{+\left[\omega_{1}, \omega_{2}\right]}^{\infty}$.

\section{Proof of Lemma 5}

Proof of Lemma 5: “ $\Leftarrow$ ”: Since

$$
|(T f)(t)| \leq \int_{-\infty}^{\infty}|f(\tau) h(t-\tau)| \mathrm{d} \tau \leq\|f\|_{\infty}\|h\|_{1}
$$

for all $t \in \mathbb{R}$ and all $f \in \mathcal{B}_{\pi, 0}^{\infty}$, it follows that

$$
\|T\|_{\mathrm{BIBO}_{\pi}}=\sup _{\substack{f \in \mathcal{B}_{\pi, 0}^{\infty} \\\|f\|_{\mathcal{B}_{\pi, 0}^{\infty} \leq 1}^{\infty} \leq 1}}\|T f\|_{\infty} \leq\|h\|_{1} .
$$

“ $\Rightarrow$ ”: Let $\omega_{1}>\pi$ be arbitrary but fixed. Since $|(T f)(0)| \leq$ $\|T f\|_{\infty} \leq\|T\|_{\mathrm{BIBO}_{\pi}}\|f\|_{\infty}$, for all $f \in \mathcal{B}_{\pi, 0}^{\infty}$, we see that the linear functional $\Phi: \mathcal{B}_{\pi, 0}^{\infty} \rightarrow \mathbb{C}, f \mapsto(T f)(0)$ is bounded. Since $\mathcal{B}_{\pi, 0}^{\infty}$ is a subspace of $C_{0}(\mathbb{R})$, we know from the HahnBanach theorem [13, p. 104] that there exists a bounded linear functional $\Psi: C_{0}(\mathbb{R}) \rightarrow \mathbb{C}$ with $\Phi f=\Psi f$ for all $f \in \mathcal{B}_{\pi, 0}^{\infty}$. Moreover, according to the Riesz representation theorem [13, p. 130], the functional $\Psi: C_{0}(\mathbb{R}) \rightarrow \mathbb{C}$ has the representation

$$
\Psi f=\int_{-\infty}^{\infty} f(\tau) \mathrm{d} u(\tau)
$$

where the integral is a Stieltjes integral and $u$ is a function of bounded variation on $\mathbb{R}$. Hence, for all $f \in \mathcal{B}_{\pi, 0}^{\infty}$ we have

$$
\Phi f=\Psi f=\int_{-\infty}^{\infty} f(\tau) \mathrm{d} u(\tau) .
$$

Let $f \in \mathcal{B}_{\pi, 0}^{\infty}$ and $t \in \mathbb{R}$ be arbitrary but fixed, and consider the function $f_{t}(\tau)=f(\tau-t)$. Due to the time-invariance of $T$ we have $(T f)(t)=\left(T f_{-t}\right)(0)$. It follows that

$$
\begin{aligned}
(T f)(t) & =\left(T f_{-t}\right)(0)=\Phi f_{-t}=\Psi f_{-t} \\
& =\int_{-\infty}^{\infty} f_{-t}(\tau) \mathrm{d} u(\tau) \\
& =\int_{-\infty}^{\infty} f(\tau+t) \mathrm{d} u(\tau) \\
& =\int_{-\infty}^{\infty} f(\tau) \mathrm{d} u(\tau-t) .
\end{aligned}
$$


Next, we show that $T f \in \mathcal{B}_{\pi}^{\infty}$. Since $T$ is assumed to be $\mathrm{BIBO}_{\pi}$-stable, we have $T f \in L^{\infty}(\mathbb{R})$. It remains to show that $T f \in \mathcal{B}_{\pi}$. Let $\Gamma$ be an arbitrary closed contour in $\mathbb{C}$. Since

$$
\oint_{\Gamma} \int_{-\infty}^{\infty}|f(\tau+z)| \mathrm{d} u(\tau) \mathrm{d} z \leq M V(u) L
$$

where $M$ is the supremum of $|f(z)|$ in some horizontal strip containing $\Gamma, L$ is the length of $\Gamma$, and $V(u)$ denotes the total variation of $u$, we can apply Fubini's theorem to obtain

$$
\begin{aligned}
\oint_{\Gamma}(T f)(z) \mathrm{d} z & =\oint_{\Gamma} \int_{-\infty}^{\infty} f(\tau+z) \mathrm{d} u(\tau) \mathrm{d} z \\
& =\int_{-\infty}^{\infty} \oint_{\Gamma} f(\tau+z) \mathrm{d} z \mathrm{~d} u(\tau)
\end{aligned}
$$

Moreover, the integral $\oint_{\Gamma} f(\tau+z) \mathrm{d} z$ is zero, because $f$ is an entire function. Thus, we have $\oint_{\Gamma}(T f)(z) \mathrm{d} z=0$ for all contours $\Gamma$ in $\mathbb{C}$, and it follows from Morea's theorem [11, p. 74] that $(T f)(z)$ is an entire function. Additionally, we have

$$
\begin{aligned}
|(T f)(z)| & \leq \sup _{\tau \in \mathbb{R}} \mid f(\tau+i \operatorname{Im}(z) \mid V(u) \\
& \leq\|f\|_{\infty} \mathrm{e}^{\pi \operatorname{Im}(z)} V(u),
\end{aligned}
$$

where we used the fact that $f \in \mathcal{B}_{\pi, 0}^{\infty} \subset \mathcal{B}_{\pi}^{\infty}$ and Lemma 1 in the last inequality. Thus, $T f \in \mathcal{B}_{\pi}$ and consequently $T f \in \mathcal{B}_{\pi}^{\infty}$.

Consider the function $g \in \mathcal{B}_{\omega_{1}}^{1}$ defined by

$$
g(t)=\frac{1}{2 \pi} \int_{-\omega_{1}}^{\omega_{1}} \hat{g}(\omega) \mathrm{e}^{i \omega t} \mathrm{~d} \omega
$$

where

$$
\hat{g}(\omega)= \begin{cases}1, & |\omega| \leq \pi \\ 1-\frac{|\omega|-\pi}{\omega_{1}-\pi} & \pi<|\omega| \leq \omega_{1} .\end{cases}
$$

Since $T f \in \mathcal{B}_{\pi}^{\infty}$, we have the identity

$$
(T f)(t)=\int_{-\infty}^{\infty}(T f)\left(\tau_{1}\right) g\left(t-\tau_{1}\right) \mathrm{d} \tau_{1}
$$

and consequently

$$
\begin{aligned}
(T f)(t) & =\int_{-\infty}^{\infty} \int_{-\infty}^{\infty} f(\tau) \mathrm{d} u\left(\tau-\tau_{1}\right) g\left(t-\tau_{1}\right) \mathrm{d} \tau_{1} \\
& =\int_{-\infty}^{\infty} f(\tau) \mathrm{d}_{\tau} \int_{-\infty}^{\infty} u\left(\tau-\tau_{1}\right) g\left(t-\tau_{1}\right) \mathrm{d} \tau_{1} \\
& =\int_{-\infty}^{\infty} f(\tau) \underbrace{\int_{-\infty}^{\infty}-u\left(\tau_{1}\right) g^{\prime}\left(\tau_{1}+t-\tau\right) \mathrm{d} \tau_{1}}_{=: h(t-\tau)} \mathrm{d} \tau
\end{aligned}
$$

The interchange of the integration order is justified by Fubini's theorem [16], because

$$
\int_{-\infty}^{\infty} \int_{-\infty}^{\infty}|f(\tau)|\left|\mathrm{d} u\left(\tau-\tau_{1}\right)\right|\left|g\left(t-\tau_{1}\right)\right| \mathrm{d} \tau_{1} \leq\|f\|_{\infty} V(u)\|g\|_{1}
$$$$
<\infty
$$

Thus, we have

$$
(T f)(t)=\int_{-\infty}^{\infty} f(\tau) h(t-\tau) \mathrm{d} \tau
$$

for all $f \in \mathcal{B}_{\pi, 0}^{\infty}$ and all $t \in \mathbb{R}$.

It remains to show that $h \in \mathcal{B}_{\omega_{1}}^{1}$. Integration by parts gives

$$
h(t)=\int_{-\infty}^{\infty}-u\left(\tau_{1}\right) g^{\prime}\left(\tau_{1}+t\right) \mathrm{d} \tau_{1}=\int_{-\infty}^{\infty} g\left(\tau_{1}+t\right) \mathrm{d} u\left(\tau_{1}\right)
$$

because $u$ is bounded and $\lim _{|t| \rightarrow \infty} g(t)=0$. Thus, it follows that

$$
\begin{aligned}
\int_{-\infty}^{\infty}|h(t)| \mathrm{d} t & =\int_{-\infty}^{\infty}\left|\int_{-\infty}^{\infty} g\left(\tau_{1}+t\right) \mathrm{d} u\left(\tau_{1}\right)\right| \mathrm{d} t \\
& \leq \int_{-\infty}^{\infty} \int_{-\infty}^{\infty}\left|g\left(\tau_{1}+t\right)\right| \mathrm{d} t\left|\mathrm{~d} u\left(\tau_{1}\right)\right| \\
& =V(u)\|g\|_{1}<\infty
\end{aligned}
$$

which shows that $h \in L^{1}(\mathbb{R})$. Using the same steps as before, where we showed that $T f \in \mathcal{B}_{\pi}$, it follows that $h \in \mathcal{B}_{\omega_{1}}$, and consequently that $h \in \mathcal{B}_{\omega_{1}}^{1}$.

\section{E. Proof of Observation 1}

Proof of Observation 1: For convenience, we introduce the set $\mathcal{U}=\left\{h \in L^{1}(\mathbb{R}): \hat{h}(\omega)=0\right.$ for all $\left.\omega \in \mathbb{R} \backslash\left(\omega_{1}, \omega_{2}\right)\right\}$.

" $\Rightarrow$ ": Let $h \in \mathcal{U}$ be arbitrary but fixed. Since $h \in L^{1}(\mathbb{R})$ and $\hat{h}(\omega)=0$ for all $\omega \in \mathbb{R} \backslash\left(\omega_{1}, \omega_{2}\right)$, it follows that $\hat{h}$ is continuous and in $L^{2}(\mathbb{R})$, and consequently we have $h \in L^{2}(\mathbb{R})$. By using Parseval's theorem twice, we obtain

$$
\begin{aligned}
\int_{-\infty}^{\infty} f(\tau) h(\tau) \mathrm{d} \tau & =\frac{1}{2 \pi} \int_{\omega_{1}}^{\omega_{2}} \hat{f}(-\omega) \hat{h}(\omega) \mathrm{d} \omega \\
& =\frac{1}{2 \pi} \int_{\omega_{1}}^{\omega_{2}} \hat{g}(-\omega) \hat{h}(\omega) \mathrm{d} \omega \\
& =\int_{-\infty}^{\infty} g(\tau) h(\tau) \mathrm{d} \tau .
\end{aligned}
$$

" $\Leftarrow$ ": Since

$$
\begin{aligned}
\frac{1}{2 \pi} \int_{\omega_{1}}^{\omega_{2}} \hat{f}(-\omega) \hat{h}(\omega) \mathrm{d} \omega & =\int_{-\infty}^{\infty} f(\tau) h(\tau) \mathrm{d} \tau \\
& =\int_{-\infty}^{\infty} g(\tau) h(\tau) \mathrm{d} \tau \\
& =\frac{1}{2 \pi} \int_{\omega_{1}}^{\omega_{2}} \hat{g}(-\omega) \hat{h}(\omega) \mathrm{d} \omega
\end{aligned}
$$

for all $h \in \mathcal{U}$, it follows that

$$
\int_{\omega_{1}}^{\omega_{2}}[\hat{f}(-\omega)-\hat{g}(-\omega)] \hat{h}(\omega) \mathrm{d} \omega=0
$$


for all $h \in \mathcal{U}$. Furthermore, since $\left(C_{0}^{\infty}\left[\omega_{1}, \omega_{2}\right],\|\cdot\|_{L^{2}\left[\omega_{1}, \omega_{2}\right]}\right)$ is dense in $L^{2}\left[\omega_{1}, \omega_{2}\right]$ and $\hat{f}-\hat{g} \in L^{2}\left[\omega_{1}, \omega_{2}\right]$, there exists a sequence $\left\{\hat{h}_{k}\right\}_{k \in \mathbb{N}} \subset C_{0}^{\infty}\left[\omega_{1}, \omega_{2}\right]$ with

$$
\lim _{k \rightarrow \infty}\left\|\hat{h}_{k}-\hat{\eta}\right\|_{L^{2}\left[\omega_{1}, \omega_{2}\right]}=0
$$

where $\hat{\eta}(\omega)=\overline{\hat{f}(-\omega)-\hat{g}(-\omega)}$. Moreover, we have

$$
\int_{\omega_{1}}^{\omega_{2}}[\hat{f}(-\omega)-\hat{g}(-\omega)] \hat{h}_{k}(\omega) \mathrm{d} \omega=0
$$

for all $k \in \mathbb{N}$, because $C_{0}^{\infty}\left[\omega_{1}, \omega_{2}\right] \subset\{\hat{f}: f \in \mathcal{U}\}$, and consequently

$$
\lim _{k \rightarrow \infty} \int_{\omega_{1}}^{\omega_{2}}[\hat{f}(-\omega)-\hat{g}(-\omega)] \hat{h}_{k}(\omega) \mathrm{d} \omega=0 .
$$

Furthermore, we have

$$
\begin{aligned}
\mid \int_{\omega_{1}}^{\omega_{2}} & {[\hat{f}(-\omega)-\hat{g}(-\omega)] \hat{h}_{k}(\omega) \mathrm{d} \omega } \\
& \quad-\int_{\omega_{1}}^{\omega_{2}}[\hat{f}(-\omega)-\hat{g}(-\omega)] \hat{\eta}(\omega) \mathrm{d} \omega \mid \\
& =\left|\int_{\omega_{1}}^{\omega_{2}}[\hat{f}(-\omega)-\hat{g}(-\omega)]\left(\hat{h}_{k}(\omega)-\hat{\eta}(\omega)\right) \mathrm{d} \omega\right| \\
& \leq\|\hat{f}-\hat{g}\|_{L^{2}\left[\omega_{1}, \omega_{2}\right]}\left\|\hat{h}_{k}-\hat{\eta}\right\|_{L^{2}\left[\omega_{1}, \omega_{2}\right]}
\end{aligned}
$$

which implies that

$$
\begin{aligned}
& \left|\int_{\omega_{1}}^{\omega_{2}}[\hat{f}(-\omega)-\hat{g}(-\omega)] \hat{\eta}(\omega) \mathrm{d} \omega\right| \leq\|\hat{f}-\hat{g}\|_{L^{2}\left[\omega_{1}, \omega_{2}\right]} \\
& \times\left\|\hat{h}_{k}-\hat{\eta}\right\|_{L^{2}\left[\omega_{1}, \omega_{2}\right]}+\left|\int_{\omega_{1}}^{\omega_{2}}[\hat{f}(-\omega)-\hat{g}(-\omega)] \hat{h}_{k}(\omega) \mathrm{d} \omega\right| .
\end{aligned}
$$

From (34) and (35) we see that the RHS of (36) tends to zero as $k \rightarrow \infty$. Therefore, we have

$$
\int_{\omega_{1}}^{\omega_{2}}|\hat{f}(-\omega)-\hat{g}(-\omega)|^{2} \mathrm{~d} \omega=\int_{\omega_{1}}^{\omega_{2}}[\hat{f}(-\omega)-\hat{g}(-\omega)] \hat{\eta}(\omega) \mathrm{d} \omega=0
$$

which implies that $\hat{f}(\omega)=\hat{g}(\omega)$ a.e. in $\left[\omega_{1}, \omega_{2}\right]$.

\section{REFERENCES}

[1] G. Wunder and H. Boche, "Peak value estimation of bandlimited signals from their samples, noise enhancement, and a local characterization in the neighborhood of an extremum," IEEE Trans. Signal Process., vol. 51, no. 3, pp. 771-780, Mar. 2003.

[2] M. Schetzen, Linear Time-Invariant Systems. New York: Wiley, 2002.
[3] B. F. Logan, Jr., "Signals designed for recovery after clipping-I. Localization of infinite products," AT\&T Bell Lab. Tech. J., vol. 63, no. 2, pp. 261-285, Feb. 1984

[4] R. P. Boas, Jr., "Some theorems on Fourier transforms and conjugate trigonometric integrals," Trans. Amer. Math. Soc., vol. 40, no. 2, pp. 287-308, 1936

[5] B. Y. Levin, Lectures on Entire Functions. New York: AMS, 1996.

[6] M. Zakai, "Band-limited functions and the sampling theorem," Inf. Contr., vol. 8, no. 2, pp. 143-158, 1965.

[7] G. Valiron, "Sur la formule d'interpolation de Lagrange," Bulletin des Sci. Math., vol. 49, no. 2, pp. 181-192, 1925, 203-224.

[8] P. L. Butzer, W. Splettstößer, and R. L. Stens, "The sampling theorem and linear prediction in signal analysis," Jahresbericht Deutschen Math.-Vereinigung, vol. 90, no. 1, pp. 1-70, Jan. 1988.

[9] J. R. Higgins, Sampling Theory in Fourier and Signal Analysis—Foundations. Oxford, U.K.: Oxford Univ. Press, 1996.

[10] A. Zygmund, Trigonometric Series, 3rd ed. Cambridge, U.K.: Cambridge Univ. Press, 2002, vol. I.

[11] R. E. Greene and S. G. Krantz, Function Theory of One Complex Variable. New York: Wiley, 1997.

[12] B. F. Logan, Jr., "Theory of analytic modulation systems," Bell System Tech. J., vol. 57, no. 3, pp. 491-576, Mar. 1978.

[13] W. Rudin, Real and Complex Analysis, 3rd ed. New York: McGrawHill, 1987.

[14] K. Yosida, Functional Analysis. New York: Springer-Verlag, 1971.

[15] L. V. Kantorovich and G. P. Akilov, Functional Analysis in Normed Spaces. New York: Pergamon, 1964.

[16] R. H. Cameron and W. T. Martin, "An unsymmetric Fubini theorem," Bull. Amer. Math. Soc., vol. 47, no. 2, pp. 121-125, 1941.

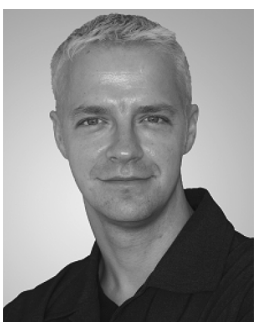

Holger Boche (M'04-SM'07) received the Dipl.-Ing. and Dr.-Ing. degrees in electrical engineering from the Technische Universität Dresden (TU), Germany, in 1990 and 1994, respectively. In 1992, he received the degree in mathematics from the TU Dresden, and in 1998, he received the Dr.rer.nat. degree in pure mathematics from the TU Berlin, Germany.

From 1994 to 1997, he did postgraduate studies in mathematics at the Friedrich-Schiller Universität Jena, Germany. In 1997, he joined the Heinrich-Hertz-Institut (HHI) für Nachrichtentechnik Berlin. Since 2002, he has been a Full Professor for Mobile Communication Networks, TU Berlin, Institute for Communications Systems. In 2003, he became Director of the Fraunhofer German-Sino Lab for Mobile Communications, Berlin, and since 2004, he has also been Director of the Fraunhofer Institute for Telecommunications, HHI, Berlin. He was Visiting Professor with the ETH Zurich during winter term 2004 and 2006 and with KTH Stockholm during summer term 2005.

Prof. Boche received the Research Award "Technische Kommunikation" from the Alcatel SEL Foundation in October 2003, the "Innovation Award" from the Vodafone Foundation in June 2006, and the Gottfried Wilhelm Leibniz Prize from the Deutsche Forschungsgemeinschaft (German Research Foundation) in 2008. He was a corecipient of the 2006 IEEE Signal Processing Society Best Paper Award and recipient of the 2007 IEEE Signal Processing Society Best Paper Award. He is a member of the IEEE Signal Processing Society SPCOM and SPTM Technical Committee. He was elected a member of the German Academy of Sciences (Leopoldina) in 2008.

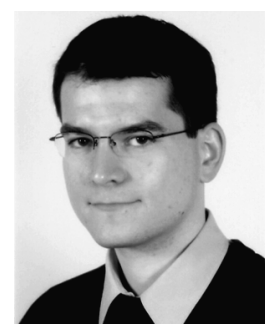

Ullrich J. Mönich (S'06) received the Dipl.-Ing. degree in electrical engineering from the Technische Universität Berlin, Germany, in 2005, where he is currently pursuing the Ph.D. degree.

During winter term 2003, he was a Visiting Researcher with the University of California, Santa Barbara. His research activities comprise sampling theory, signal processing, and information theory. 


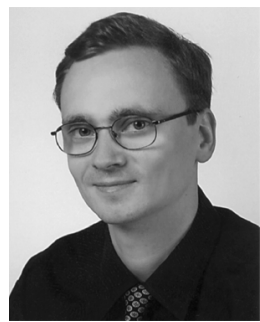

Andreas Kortke received the Dipl.-Ing. and the Dr.Ing. degrees in electrical engineering from the Technische Universität (TU) Berlin, Germany, in 1999 and 2006, respectively.

$\mathrm{He}$ is with the Heinrich-Hertz-Chair for Mobile Communications of the TU Berlin and with the Broadband Mobile Communication Networks Department, Fraunhofer Heinrich-Hertz-Institute, Berlin. His research interests are in wireless communication systems, RF engineering, and digital signal processing.

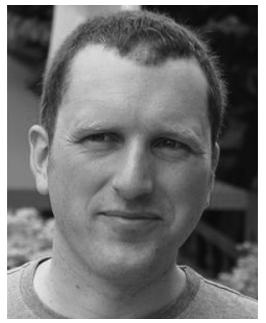

Wilhelm Keusgen was born in Rheinbach, Germany, in 1973. He received the Dipl.-Ing. (M.S.E.E.) and Dr.-Ing. (Ph.D.E.E.) degrees from the RWTH Aachen University, Aachen, Germany, in 1999 and 2005, respectively.

From 1999 to 2004, he was with the Institute of High Frequency Technology, RWTH Aachen University, where he worked on multiple antenna systems, microwave front-end technologies and microwave antennas. In 2004, he joined the Fraunhofer Institute for Telecommunications, Heinrich Hertz Institute located, Berlin. He currently leads a research group that is focused on the design and implementation of broadband mobile communication systems. His main research areas are multiple antenna systems, broadband millimeter wave communication, car-to-car communication, and digital compensation of RF impairments especially for power amplifiers. 